Portland State University

PDXScholar

Fall 11-2-2016

\title{
The Application of Electrochemical Impedance Spectroscopy to Immediately Diagnose the Protective Quality of Coatings on Artistic and Architectural Metalwork
}

Kathryn Nicole Hosbein

Portland State University

Follow this and additional works at: https://pdxscholar.library.pdx.edu/open_access_etds

Part of the Chemistry Commons

Let us know how access to this document benefits you.

\section{Recommended Citation}

Hosbein, Kathryn Nicole, "The Application of Electrochemical Impedance Spectroscopy to Immediately Diagnose the Protective Quality of Coatings on Artistic and Architectural Metalwork" (2016). Dissertations and Theses. Paper 3324.

https://doi.org/10.15760/etd.3305

This Thesis is brought to you for free and open access. It has been accepted for inclusion in Dissertations and Theses by an authorized administrator of PDXScholar. Please contact us if we can make this document more accessible: pdxscholar@pdx.edu. 
The Application of Electrochemical Impedance Spectroscopy to Immediately

Diagnose the Protective Quality of Coatings on Artistic and Architectural Metalwork

by

Kathryn Nicole Hosbein

A thesis submitted in partial fulfillment of the

requirements for the degree of

Master of Science

in

Chemistry

Thesis Committee:

Tami Lasseter Clare, Chair

Dean Atkinson

Erik Johansson

Portland State University

2016 
(C) 2016 Kathryn Nicole Hosbein 


\begin{abstract}
Corrosion is a spontaneous process that causes irreversible damage to nearly all metals, which has world-wide implications for architectural and artistic metalwork, such as bridges, buildings, airplanes and sculptures. Protective coatings such as wax, paint, or polymeric clear coatings are used to prolong the lifetime of metals such as steel and bronze. Unfortunately, these coatings fail over time due to oxidative damage by UV rays and by failure to exclude water that can carry salts and pollutants that cause corrosion of the underlying substrate. The current method of coating assessment is visual inspection but when coating failure is detected at this stage, irreversible damage has already occurred to the metal substrate. Diagnosing coating quality on artistic metalwork is a unique challenge as it requires a method that is non-destructive as to not alter the aesthetic of the piece. A non-destructive technique or device that can detect early signs of coating failure in the field (such as at a sculpture park) does not currently exist. The aim of this thesis is to develop a method that can be readily used in the field by a conservator to quickly diagnose the protective state of a coating on a sculpture in order to provide localized treatment.

Electrochemical impedance spectroscopy (EIS) is a method currently used to study protective coatings in the lab. The technique itself is non-destructive but the most common electrochemical cell used with it must be used on a planar substrate and requires that a portion of coating be removed. Not only is the current method destructive, but the data produced by EIS is complicated and time consuming to analyze. These issues provide the foundation for this project.
\end{abstract}


This thesis first proposes multiple methods to quickly analyze the complicated data traditionally collected through EIS. Three quick analysis methods, including the estimation of the derivative at one single frequency in EIS spectra, was successfully used to categorize coating quality of five different coating types. Using this quick analysis can aid conservators in assessing coating condition without the need for extensive training in EIS data interpretation.

This thesis also proposes a method to measure early warning signs of coating degradation through a co-planar hydrogel electrochemical cell paired with EIS. The configuration of this co-planar hydrogel cell negates the need for the removal of the coating and can be used on multiple types of surfaces because of its flexibility, therefore overcoming the drawbacks of the traditional EIS electrochemical cell. Data provided demonstrates that this co-planar hydrogel provides similar information, when compared to the standard electrochemical cell, about the bulk of the protective coating. Unique to the co-planar hydrogel cell, information about surface degradation is provided during EIS measurements. This provides a warning sign before bulk degradation of the coating and therefore before any damage to the underlying substrate has occurred. 


\section{Acknowledgements}

This journey has been one of the most challenging and rewarding I've ever faced. It would have been much shorter without the help of some very influential people. First and foremost, my adviser Dr. Tami Lasseter Clare, who pushed me further than I have ever been. This led to invaluable lessons about persistence and motivation as well as revelations about myself. I sincerely thank you.

To my committee and other supportive faculty members, thank you for the time you have given me so that I could grow into the scientist and educator I am today. To Dr. Erik Johansson, for a contribution to the essential electrochemical knowledge I needed to persevere. To Dr. Dean Atkinson, for giving me the freedom to explore teaching in a way that led to the discovery of my passion for education. To Dr. Gwen Shusterman for opening your door to me when I needed it the most. To Dr. Jack Barbera for your generous support and introducing me to the world of chemical education.

To my friends at Portland State, thank you for the community. Specifically, thank you to two of my lab mates, Capri Price and Dr. Natasja Swartz, for all of the input on and contribution to this work as well as the intellectual and philosophical conversations. You two were my anchors throughout this process. Thank you as well to Dr. Alice England for all of the lifelong advice during your time here.

To my mother, Kristin, who helped me move to Portland and has been there in crucial times of need. To my father and step-mother, D.J. and Renée, for always calling to check-in and make sure that I was staying sane and keeping on track. To my brother and sister-in-law, Ben and Jessica, for always being available to talk to when I 
needed a distraction. To my cousins, Annie and Dave, and best friends, Josh, Tulo, and Kristin for making the time for me during my short visits back to the Midwest. To my oldest friend, Christie, and Brian for moving across the country to live with Eric and me and for providing unwavering support. Thank you all. You may now refer to me as Master Katy.

And lastly, to Eric — without all of your love and laughs, I would have never made it through. 


\section{Table of Contents}

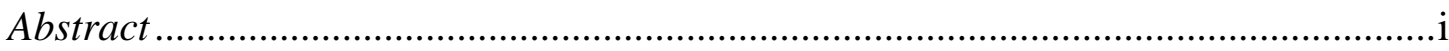

Acknowledgements ....................................................................................... iii

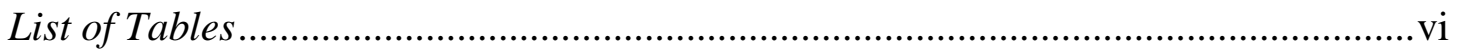

List of Figures ...............................................................................................

1 Electrochemical identification and categorization of the protective quality of intact

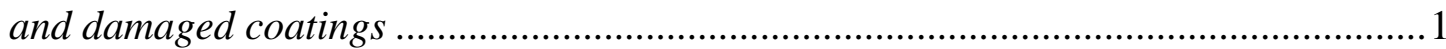

2 Understanding lateral pathway measurements by co-planar hydrogel

electrochemical cells for characterizing organic layers on surfaces ........................26

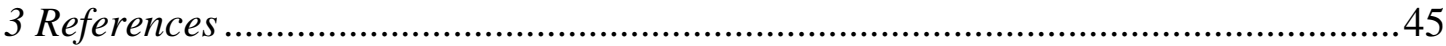

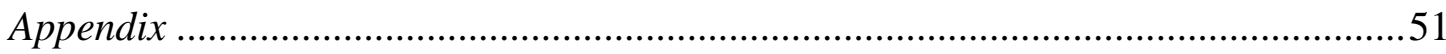




\section{List of Tables}

Table 1.1 Values of parameters for Quick Analysis Methods.................................... 15

Table 1.2. Values of parameters for Quick Analysis Methods continued .................... 16 


\section{List of Figures}

Figure 1.1 EEC models used in fitting the EIS spectra. .............................................

Figure 1.2 Two general classes of polymer coating failure .....................................4

Figure 1.3 EIS spectra and EEC model of an abraded Paraloid ${ }^{T M}$ B-44 coating on bronze 10

Figure 1.4 EIS spectra of a Paraloid ${ }^{T M}$ B-44 coating on bronze with multiple point defects 11

Figure 1.5 EIS spectra and EEC models of Paraloid ${ }^{T M}$ B-44 coatings on bronze before and after various damage.... 13

Figure 1.6 Correlation plots of quick analysis methods vs. EEC classification of various coated metal panels 19

Figure 1.7 A An approximation of the first derivative by parabolic fitting of EIS spectra of various coated metal panels

Figure 2.1 EIS spectra and EEC models of TEC ${ }^{T M} 250$ glass with varied co-planar cell geometry.

Figure 2.2 Changes in resistance with varied co-planar cell geometry on functionalized soda lime glass surfaces 34

Figure 2.3 Model fits and sheet resistance of functionzlied soda lime and Optiwhite ${ }^{T M}$ glass surfaces 36

Figure 2.4 Standard cell and co-planar cell EIS spectra and SEM images of primer and paint after natural and artificial weathering..... 41

Figure 2.5 A Standard cell and co-planar EIS spectra of Tony Smith's Stinger........ 43

Figure 2.6 Sheet resistances of weathered primer, paint, and Tony Smith's Stinger. .45 


\section{Electrochemical identification and categorization of the protective quality of intact and damaged coatings}

Adapted with permission from: Hosbein, K. N.; Swartz N.A.; Clare, T.L., Electrochemical identification and categorization of the protective quality of intact and damaged coatings. Electroanalysis 2014

Defective polymeric coatings that are particularly relevant in the conservation of outdoor metalwork, are analyzed using electrochemical impedance spectroscopy (EIS), validated by Kramers-Kronig transformations, and modeled using electrical equivalent circuit models (EECS). Using twenty different coated panels of five different coating types, ten mathematical methods for categorizing the protective qualities of coatings are explored as simpler and faster alternatives to circuit modeling; three methods gave a perfect correlation with the category determined by circuit modeling. Our findings highlight the need for fitting data to EECs before relying upon purely mathematical parameters for evaluating protective coating quality.

\subsection{Introduction}

Protective coatings are often applied to outdoor metalwork such as sculptures, architectural, and structural elements to prevent corrosion and/or preserve the underlying patina. To avoid irreversible damage to such unique works or critical components, it is crucial to detect the early signs of coating failure and to do so using nondestructive methods. Electrical sensing methods are increasingly under investigation as they offer the possibility for real-time and continuous monitoring ${ }^{1}$. Over recent decades, electrochemical impedance spectroscopy (EIS) has been utilized 
in research initiatives to investigate the barrier properties of these coatings and their effectiveness at preventing corrosion on various substrates ${ }^{2-3}$. This diagnostic technique can be used to monitor changes in protective coatings without causing damage to the underlying metal substrate as measurements can be made at the open circuit potential (no net current), allowing repeated measurements to be made over time without alteration of the substrate ${ }^{4}$. The barrier properties of a coating system are not only determined by the dielectric properties of the film, but also by mechanical deformations that occur during application or damage accrued during the coating's lifetime 5 . Properties of coating failure may include water uptake, thinning, or delamination of the coating from the metal substrate, all of which may give measurable changes in the impedance spectra.

To provide quantitative insight to the system, impedance spectra are often interpreted by fitting the data to equivalent electrical circuit models (EECs) that represent the physical system being analyzed. A typical circuit for a polymeric film on metal is shown in Figure 1.1, labeled EEC 1. The $R C$ time constant is the product of a resistor and capacitor in parallel; and as such occurs at $-45^{\circ}$ in phase with units in time $\left(\mathrm{Hz} \mathrm{or} \mathrm{s}^{-1}\right)$ where it is used to represent the time required for charge to travel through the coating to the metal working electrode. When calculated from impedance spectra of a coating having defects, the time constant that appears at the highest frequencies $\left(T_{1}=R_{\text {pore }} \cdot C_{\text {coat }}\right)$ may represent the resistance of pores within the coating and the capacitance of the coating. If present, the second time constant at lower frequencies may also represent the coating where some portion differs largely from that modeled by the first $\left(T_{2}=R_{\text {pore } 2} \cdot C_{\text {coat } 2}\right)$ as is the case for an electrolyte saturated layer in EEC $2^{6-}$ 
7. However, in most cases, the working area of the coating being examined is not a bilayer and the second time constant (when observed) is indicative of the electrical double-layer that forms on any exposed metal surface, represented by a charge transfer resistance and double layer capacitance $\left(T_{2}=R_{c t} \cdot C_{d l}\right)^{8}$. When corrosion is present on the metal surface, three time constants may be observed, as seen in EEC 3, and can be attributed to an oxide layer on the metal surface $\left(T_{3}=R_{o x} \cdot C_{o x}\right)^{7}$. When fitting experimental data, often constant phase elements (CPEs) are used in place of ideal capacitors $^{9}$. The EEC models fit to impedance spectra provide numerical values for different circuit elements that can be monitored over time for weathering-induced changes.

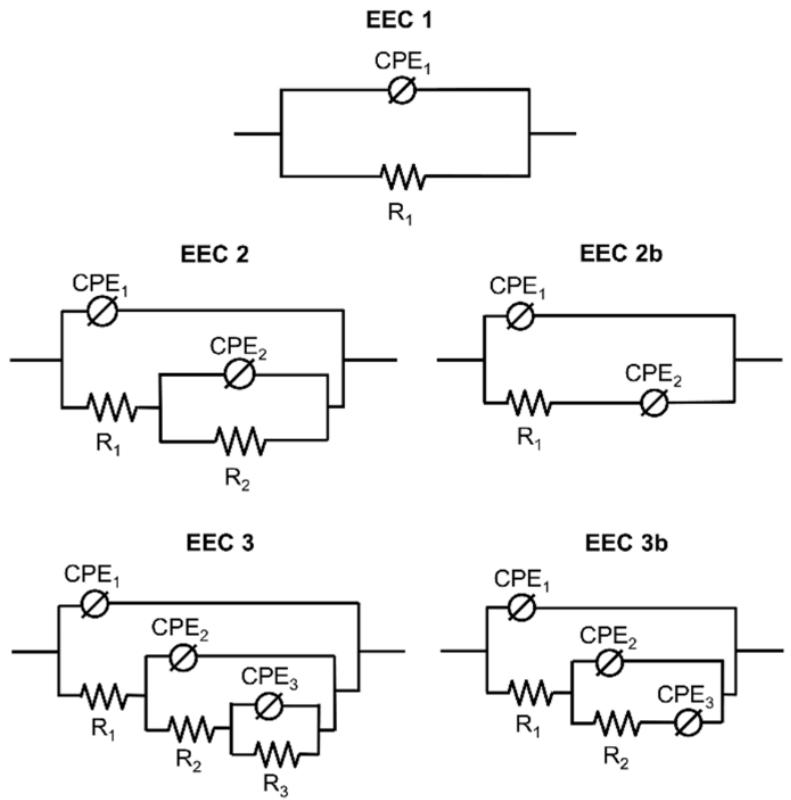

Figure 1.1 EEC models used in fitting the EIS spectra.

When mechanical damage is intentionally applied to a coating system and then monitored by EIS, the analyzed spectra can be used to explain similar processes observed in weathered samples $5,7,10-11$. Although there are numerous types of mechanical defects that may occur in a protective coating, two general classes of failure 
are depicted in Figure 1.2: abrasion and pinhole defects (i.e. film thickness loss and exaggerated pores). In this work, we have mechanically altered solvent based polymer films on bronze substrates in an effort to emulate the EIS features observed in films weathered outdoors in Portland, OR, South Florida, and by accelerated QUV-B methods. Through EIS data analysis, we aim to determine the electrochemical signatures of damage to naturally weathered, accelerated weathered and intentionally damaged films.

A)

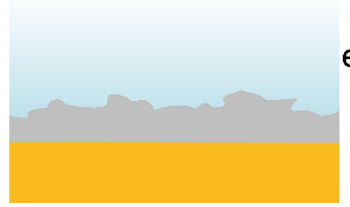

B)

$$
\begin{gathered}
\text { electrolyte } \\
\text { coating } \\
\text { metal }
\end{gathered}
$$

Figure 1.2 Bronze panels coated with a polymer having been damaged by a) abrasion, that non-uniformly thinned the film and b) a pinhole defect, that created a pathway for direct electrolyte access to the metal substrate.

A simplified interpretation of the impedance of a coated metal states that the larger the total impedance at low frequencies (e.g. $1 \mathrm{M} \Omega$ at $0.1 \mathrm{~Hz}$ ), the greater the protective nature of the coating ${ }^{12}$. However, if the lowest frequency $R C$ pair does not represent the coating, and instead represents the oxide layer, this interpretation is misleading. Some polymeric coatings are transparent, assisting the EIS data interpretation in some cases by making underlying corrosion visible. However, if the coating is pigmented and the total impedance at low frequencies is a large value, it may be difficult to distinguish the large impedance value of a thick coating versus the presence of an oxide layer without circuit modeling ${ }^{13-15}$. While having a comprehensive electrical circuit model provides a physical representation of the system under study, 
construction and thorough interpretation of EEC models requires considerable time; thus making this method impractical for field applications.

For applications, including that of cultural heritage conservation, having quicker methods for assessing the protective quality of coatings, that do not require constructing EEC models, is desired. Simplification of the EIS data analysis will likely increase their use of impedance sensors and several methods to accomplish this have been investigated ${ }^{16}$. Some methods that have specifically been used in ranking the protective quality of coatings include: the position of the coating time constant or breakpoint frequency $\left(T_{R C}\right)^{17-18}$, single-frequency impedance ratios ${ }^{18}$, the phase angle at high frequencies ${ }^{19}$, the rate of change of either impedance modulus ${ }^{20-21}$ or the phase angle ${ }^{22}$, and the percentage of decreasing area under the Bode plot $^{23-24}$. However, many of these methods utilize only one data point in the spectrum to determine the protective quality which is potentially problematic when multiple circuit elements are present in the spectrum, as is usually the case, and when the impedance values of those elements are overlapping. For example, the time constant associated with the coating is often not clearly visible in spectra and therefore this parameter is difficult to extract without also first fitting the data to an EEC. Other methods such as the rate of change of phase or impedance or the change in area of the Bode plot utilize a large portion of the EIS spectrum. While more accurate (when successful), data collection over a large frequency range requires a great deal of time and can be impractical. We investigated alternative options for fast data analysis using the slope of the impedance magnitude, approximations of the first derivative as a function of frequency at different frequency ranges, and at single frequencies. 


\subsection{Experimental}

\subsubsection{Materials}

Coatings used were: 1) Paraloid ${ }^{\mathrm{TM}}$ B-44 (Dow, Inc) dissolved in toluene (Sigma Aldrich) 2) Incralac (StanChem formulation 69X1732), 3) Renaissance Wax (Talas) 4) Tnemec Series 27-F.C. Typoxy® and 5) Tnemec Series 175-Endura-Shield® topcoat with N69-Hi-Build Epoxoline ${ }^{\circledR}$ II primer. A Fuji HVLP Super 4 XPC $^{\mathrm{TM}}$ was used to spray coatings 1 and 2 on dry, brass or bronze substrates $(8 \mathrm{~cm} \times 15 \mathrm{~cm}, 85 \% \mathrm{Cu}, 15 \%$ Zn, TB Hagstoz \& Sons Inc or $2.4 \mathrm{~cm} \times 6 \mathrm{~cm}$, Alloy 521, US Brass and Copper) with a film thickness of $26.0 \pm 7.10 \mu \mathrm{m}$. All metal substrates were degreased with $p$-xylenes then acetone and sanded with 400 grit followed by 600 grit sandpaper prior to spraycoating to create a uniform bare metal surface. Coatings 4 and 5 were pre-painted by Tnemec on $8 \mathrm{~cm} \times 15 \mathrm{~cm}$ steel sample panels with thicknesses of $214.4 \pm 7.2$ and 117.4 $\pm 8.2 \mu \mathrm{m}$ respectively.

\subsubsection{Weathering and Intentional Damage}

Coated panels were weathered outdoors in Portland, OR, at the Q-Lab standard testing site in Homestead, Florida according to ASTM D6675 or by accelerated exposure in a QUV-B chamber (Q-Lab QUV Accelerated Weathering Tester) for 500 hours according to ASTM G154 cycle B. Intentionally damaged coatings on bronze substrates were either abraded or had multiple pinhole defects. Substrates were abraded with 1000 grit SiC sandpaper (McMaster-Carr). Coatings with multiple defects had 1 $\mathrm{cm}$ spacing between pinholes and were created using a diamond scribe. Damaged areas 
of the coatings were immersed in $3 \% \mathrm{NaCl}$ and EIS measurements were performed on the area every hour after the initial 30 minute soaking time for up to 50 hours.

\subsubsection{Electrochemical Impedance Spectroscopy}

EIS measurements were obtained using a Gamry Reference 600 Potentiostat with Gamry Framework 6 software. EIS data was acquired from $1 \mathrm{MHz}$ to $0.1 \mathrm{~Hz}$ or $0.01 \mathrm{~Hz}$ (10 points per logarithmic decade) using a standard three electrode Gamry Paint cell with a graphite counter electrode, $\mathrm{Ag} / \mathrm{AgCl}$ reference electrode, and the coated metal panel as the working electrode. The working electrode area was $14.62 \mathrm{~cm}^{2}$ with a distance of $4 \mathrm{~cm}$ between working and counter electrodes. An AC potential of $20 \mathrm{mV}_{\mathrm{rms}}$ and a DC potential of $0.0 \mathrm{~V}$ vs open circuit potential (which ranged between 200 and $-200 \mathrm{mV}$ ) was applied. Kramers-Kronig transformations were conducted to validate the EIS data and Nyquist plots and goodness of fit values are presented in the Appendix. EIS Spectra were analyzed using EEC models constructed using Gamry Echem Analyst and fit using the Simplex method. CPEs were commonly used in the models in place of capacitors to better describe the non-ideal nature of the system. Impedance of individual circuit elements were calculated for resistors:

$$
Z_{R}=R
$$

where $R$ is $\Omega \cdot \mathrm{cm}^{2}$ and for constant phase elements:

$$
Z_{C P E}=1 / Y_{0}(j \omega)^{\alpha}
$$

where $Y_{0}$ is $\mathrm{nS} \cdot \mathrm{s}^{\alpha} \cdot \mathrm{cm}^{-2}, j$ is $(-1)^{1 / 2}, \omega$ is the angular frequency, and $\alpha$ is $0 \leq \alpha \leq 1$ (an $\alpha$ of 0 is a pure resistor and an $\alpha$ of 1 is a pure capacitor). 


\subsection{Results and Discussion}

\subsubsection{Controlled damage to coated substrates: abrasion and pinholes}

Many polymeric coatings used on outdoor works are subject to thermal- and photodegradation, where smaller, often volatile, molecular fragments are produced during weathering, resulting in thinning of the coating ${ }^{25}$. In order to mimic this type of failure, a Paraloid ${ }^{\mathrm{TM}}$ B-44 coated bronze panel was mechanically abraded and measured by EIS during 50 hours of immersion in electrolyte and the spectra are overlaid in Figure 1.3. Time points were separated into two sets to better visualize spectral features: 0 to 10 hours spectra are shown in Figure $1.3 \mathrm{~A}$ and 15 to 50 hours in Figure 1.3B. In the initial spectrum at $\mathrm{t}=0$ hours a single time constant assigned to the coating $\left(T_{1}\right)$ was readily apparent at $2.0 \mathrm{~Hz}$, and shifted to higher frequencies $(10 \mathrm{~Hz})$ with increased immersion time. From 15 to 50 hours $T_{1}$ was unchanged and suggested that the pair no longer represented an active process such as water uptake. All spectra in Figure 1.3 were modeled using EEC 2b (shown in Fig. 1.1) and the values of the elements in that circuit were plotted over time in Figure 1.3C. The model was designed using both a physical interpretation of the coated system and its good fit to the data. As can be seen in Figure 1.3C the value of $C P E_{1}$, which dominated the spectrum from $1 \mathrm{kHz}$ to $1 \mathrm{MHz}$, did not change significantly during the 50 hour exposure to electrolyte. Due to its small and unchanging value (average of $0.265 \mathrm{nS} \cdot \mathrm{s}^{\alpha} \cdot \mathrm{cm}^{-2}$ ) and near ideal alpha (average of 0.967), $C P E_{1}$ corresponded to the capacitance of the intact portion of the coating $\left(C P E_{\text {coat }}\right)$. During immersion pathways having higher conductivity may be formed as electrolyte fills pores leading to a resistance, $R_{l}$, which was observed to decrease in Figure $1.3 \mathrm{C}$ from 258 to $45.0 \mathrm{M} \Omega \cdot \mathrm{cm}^{2}$, and was thus attributed to the resistance of the 
pore-like defects within the coating. $\left(R_{\text {pore }}\right)$. Similar to the trend observed in $C P E_{c o a t}$, after 10 hours of soaking the pores became saturated with electrolyte and the value of $R_{\text {pore }}$ became constant.

Abrasion caused defects to appear in the coating which were observed from 0.03 to $0.50 \mathrm{~Hz}$ as a second low frequency capacitive element. $C P E_{2}$ can be seen in EEC $2 \mathrm{~b}$ (in Fig. 1.1), which likely represents the electrical double layer $\left(C P E_{d l}\right)$. While a charge transfer resistance would be typically observed in parallel with an electrical double layer, an additional resistor was not observed over the measured frequency range and was thus not included in the model. $C P E_{2}$ was dominant at low frequencies $(\sim 20 \mathrm{mHz})$ and from 0 to 10 hours appeared to increase in magnitude $\left(2.57\right.$ to $\left.4.54 \mathrm{nS} \cdot \mathrm{s}^{\mathrm{a}} \cdot \mathrm{cm}^{-2}\right)$ and increase in phase $\left(-43.6^{\circ}\right.$ to $\left.-35.1^{\circ}\right)$ which, given its small value, provided evidence of microscopic delamination at the coating-metal interface. Visible delamination or blistering was not observed in any of these experiments and so the term delamination is used here to describe these interactions at the atomic/molecular level, rather than applied to the bulk material. The value of the $C P E_{d l}$ increased rapidly from 4.54 to 12.3 $\mathrm{nS} \cdot \mathrm{s}^{\mathrm{a}} \cdot \mathrm{cm}^{-2}$ between 10 and 15 hours. It was expected that upon immersion in electrolyte the area of the metal-electrolyte interface would expand due to delamination of the film, and this process was observed here as a sharp increase in the double layer capacitance related to an increase in area of the metal-electrolyte interface. During the period from 15 to 50 hours, the value of $C P E_{d l}$ slowly decreased. The values and fluctuations in values between 10 and 50 hours were consistent with those previously reported for double layer capacitances on top of oxide layers ${ }^{8}$. After 50 hours, micron sized spots of 
corrosion were observed. It is worth noting that an infinite Warburg element can be used in place of $C P E_{2}$, but doing so significantly decreased the quality of the model fit.

The electrochemical signature for an abraded Paraloid ${ }^{\mathrm{TM}}$ B-44 coating included a single time constant representing the coating/pore resistance and an additional capacitive element representing the double layer. The double layer was detected by EIS at the initial time point, meaning the metal-electrolyte interface was exposed at the microscopic scale. The EEC model for abrasion suggested that areas of the coating were beginning to fail after 10 hours of immersion in electrolyte due to further delamination of the film. This conclusion was supported by the presence of visible corrosion (having the appearance of small darkened spots) on the surface of the bronze substrate.
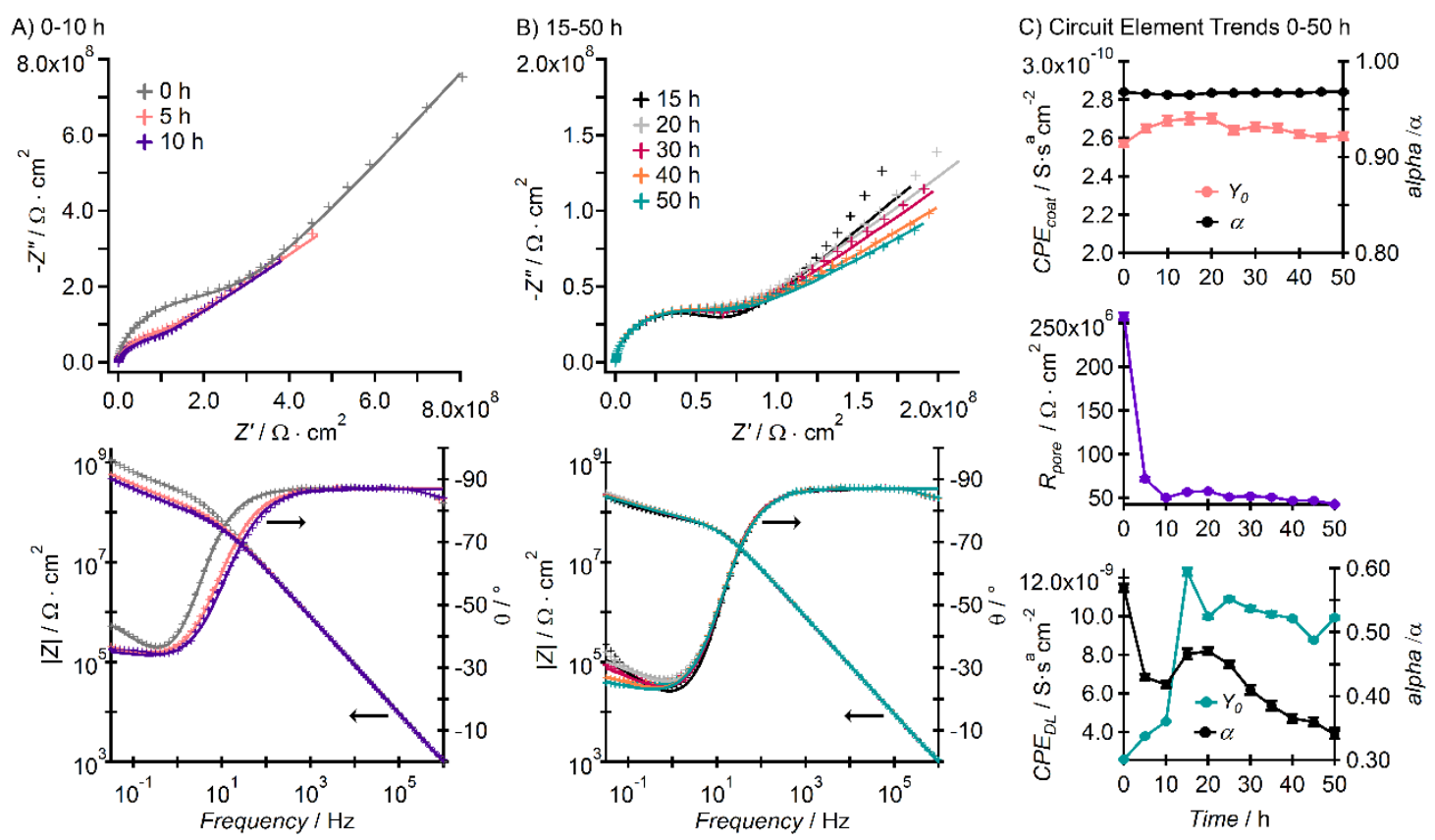

Figure 1.3 Nyquist and Bode plots of an abraded Paraloid ${ }^{\mathrm{TM}}$ B-44 coating exposed to electrolyte for A) 0-10 hours and B) 15-50 hours. Spectra shown are from every five or 10 hours during the exposure. Arrows indicate the corresponding axis for each data set. C) values of individual circuit elements from EEC model $2 \mathrm{~b}$ of an abraded polymer coating on bronze immersed in electrolyte over 50 hours: coating capacitance (top), pore resistance (middle), and double-layer capacitance (bottom). The OCP varied from -66 to $-50 \mathrm{mV}$ during the 50 hours. 


\subsubsection{Modeling and fitting impedance changes of protective films with pinhole defects}

To simulate a more severely damaged coating, multiple pinhole defects (MPD) at a $1 \mathrm{~cm}$ pitch were introduced into Paraloid ${ }^{\mathrm{TM}}$ B-44 coated substrates. Coated substrates having MPD were immersed in electrolyte for a period of 18 hours and EIS spectra acquired every hour. Spectra at selected time intervals (1, 3, 7, and 18 hours) are displayed in Figure 1.4. Identification of the physical parameters and their corresponding circuit elements was not possible in this case because there existed two partially overlapping $R$-CPE pairs in the middle region of each spectrum (between 10 $\mathrm{Hz}$ and $10 \mathrm{kHz}$, with $C P E$ maxima at approximately $0.05 \mathrm{~Hz}$ and $15 \mathrm{~Hz}$, respectively).

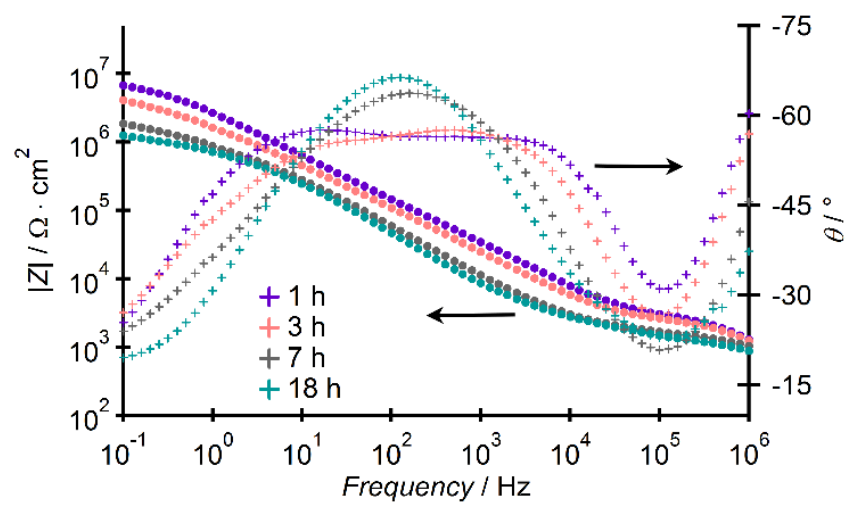

Figure 1.4 EIS spectra of a bronze panel coated with Paraloid ${ }^{\mathrm{TM}}$ B-44 having MPD over the course of immersion in electrolyte for 18 hours.

\subsubsection{Comparison between weathered and intentionally damaged protective coatings}

The intentionally damaged coatings were compared to coatings undergoing accelerated weathering to find similarities in their spectra that are indicative of degradation/damage. EIS spectra of Paraloid ${ }^{\mathrm{TM}} \mathrm{B}-44$ coated substrates (S1-S4) were acquired at the start of the experiment (before induced damage or weathering) and the data are shown in Figure 1.5A. EIS spectra of the coatings after either intentional damage was introduced (S1 and S3) or weathering (S2 and S4) are shown in Figure 
$1.5 \mathrm{~A}$ as well. The EIS spectra of S1/abraded and S2/QUV-B $500 \mathrm{~h}$ were similar both before and after either abrasion or weathering. Both samples showed one time constant $\left(T_{l}=2.0 \mathrm{~Hz}\right)$ and an additional capacitive element (maximum at $0.1 \mathrm{~Hz}$ ) where EEC $2 \mathrm{~b}$ (shown in Fig. 1.1) was used to fit to these data. Based on this model, it was apparent that pores in the coating were present and the metal/electrolyte interactions were observable by EIS. Because the values of each circuit element in the weathered and abraded coated panels of Figure 1.5B were similar, it is likely that they correspond to the same physical parts of the system. From these, we deduce that QUV-B weathered panel showed similar changes in the EIS spectrum as an abraded coated panel.

EEC 3 (Fig. 1.1) was used to fit the data from S3/MPD and S4/QUV-B 500 hours. Comparing the EIS data from S3 and S4 after the introduction of multiple point defects (S3/MPD) or weathering (S4/QUV-B 500 hours) showed that the values of the three time constants present were comparable: $T_{1}=500 \mathrm{kHz}, T_{2}=800 \mathrm{~Hz}$, and $T_{3}=1 \mathrm{~Hz}$, and suggested that similar damage occurred in those two coated panels. Because an additional CPE and resistor were seen in the EIS spectra of the S3/MPD and S4/QUVB $500 \mathrm{~h}$ samples, which were not seen in the spectra of S1/abraded and S2/QUV-B 500 hours, we concluded that corrosion processes were active for those samples. Based on the values of the individual circuit elements displayed in Figure 1.5B, the porous area of S4/QUV-B $500 \mathrm{~h}$ was smaller (having a higher resistance) than the area of the pinholes in the S3/MPD sample. Both S2 and S4 had undergone accelerated weathering for 500 hours, yet their EIS spectra were not identical. The difference was due to subtle differences in the initial quality of the film. As shown in the initial data of Figure 1.5, S2 started as a nearly ideal coating while S4 showed a small defect present indicated 
by other elements dominating the circuit at frequencies between 0.1 and $1.0 \mathrm{~Hz}$ for S2

(which was not seen in S4). Although the two coatings were weathered for the same length of time, S4 had poorer barrier properties than S2 after weathering, which caused it to fail at 500 hours of QUV-B exposure while S2 provided adequate protection.
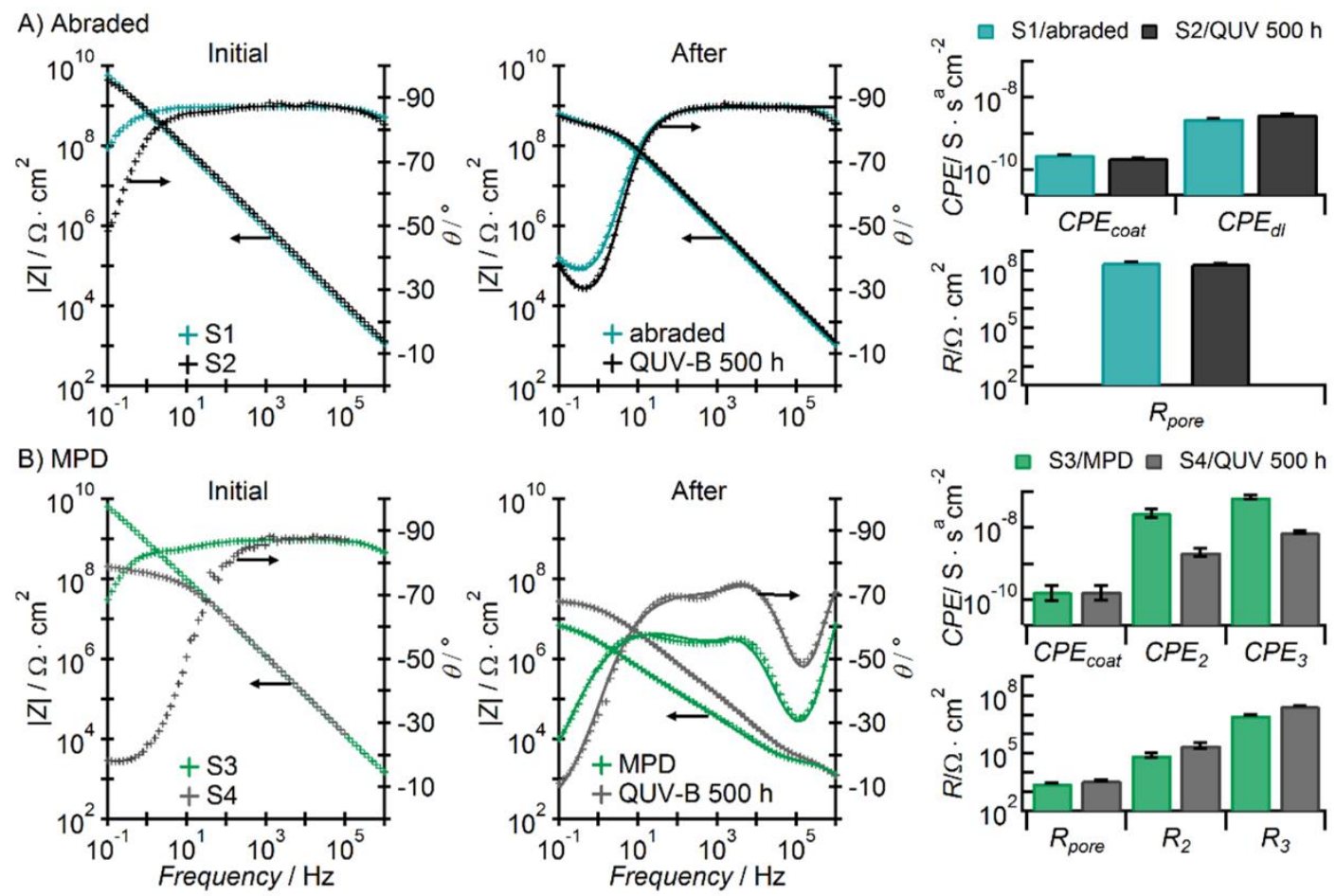

Figure 1.5 EIS data of Paraloid ${ }^{\mathrm{TM}}$ B-44 coated substrates at the start of the experiment (S1-S4) and after intentional abrasion(S1/abraded), the introduction of multiple point defects (S3/MPD) or artificial weathering (S2 or S4/QUV-B $500 \mathrm{~h}$ ). Model fit lines are displayed as the sold line traces. A) Paraloid ${ }^{\mathrm{TM}}$ B-44 coatings damaged by abrasion or artificially weathered for 500 hours in a QUV-B chamber (values reported respectively: $\mathrm{CPE}_{\text {coat }}: 2.57 \pm 0.0153 \times 10^{-10} \mathrm{~S} \cdot \mathrm{s}^{\mathrm{a}} \cdot \mathrm{cm}^{-2}, \alpha: 0.968 \pm 0.000575$ and $2.11 \pm 0.0242$ $\times 10^{-10} \mathrm{~S} \cdot \mathrm{s}^{\mathrm{a}} \cdot \mathrm{cm}^{-2}, \alpha: 0.970 \pm 0.00110 \mathrm{~S} \cdot \mathrm{s}^{\mathrm{a}} \cdot \mathrm{cm}^{-2}, \mathrm{CPE}_{\mathrm{dl}}: 2.57 \pm 0.0273 \times 10^{-9} \mathrm{~S} \cdot \mathrm{s}^{\mathrm{a}} \cdot \mathrm{cm}^{-2}, \alpha: 0.569 \pm .00602$ and $3.34 \pm 0.0810 \times 10^{-9} \mathrm{~S} \cdot \mathrm{s}^{\mathrm{a}} \cdot \mathrm{cm}^{-2}, \alpha: 0.625 \pm 0.0209$ and $\mathrm{R}_{\text {pore }}: 2.58 \pm 0.0406 \times 10^{8}$ and $2.78 \pm 0.0719$ $\times 10^{8} \Omega$. B) Paraloid ${ }^{\mathrm{TM}}$ B-44 coatings with multiple pinhole defects or artificially weathered for 500 hours in a QUV-B chamber (values reported respectively): $\mathrm{CPE}_{\text {coat }}: 1.72 \pm 0.135 \times 10^{-10} \mathrm{~S} \cdot \mathrm{s}^{\mathrm{a}} \cdot \mathrm{cm}^{-2}, \alpha$ : $0.970 \pm 0.0292$ and $2.10 \pm 0.400 \times 10^{-10} \mathrm{~S} \cdot \mathrm{s}^{\mathrm{a}} \cdot \mathrm{cm}^{-2}, \alpha: 0.97, \mathrm{CPE}_{2}: 2.54 \pm 0.657 \times 10^{-8} \mathrm{~S} \cdot \mathrm{s}^{\mathrm{a}} \cdot \mathrm{cm}^{-2}, \alpha: 0.781$ \pm 0.0225 , and $2.30 \pm 0.230 \times 10^{-9} \mathrm{~S} \cdot \mathrm{s}^{\mathrm{a}} \cdot \mathrm{cm}^{-2}, \alpha: 0.89, \mathrm{CPE}_{3}: 7.03 \pm 0.776 \times 10^{-8} \mathrm{~S} \cdot \mathrm{s}^{\mathrm{a}} \cdot \mathrm{cm}^{-2}, \alpha: 0.625 \pm$ 0.00839 , and $6.70 \pm 0.260 \times 10^{-9} \mathrm{~S} \cdot \mathrm{s}^{\mathrm{a}} \cdot \mathrm{cm}^{-2}, \alpha: 0.69, \mathrm{R}_{\text {pore }}: 2.73 \pm 0.0135 \times 10^{3}$ and $4.00 \pm 0.130 \times 10^{3}$ $\Omega, \mathrm{R}_{2}: 8.06 \pm 2.29 \times 10^{4}$ and $3.40 \pm 0.480 \times 10^{5} \Omega$, and $\mathrm{R}_{3}: 1.00 \pm 0.0392 \times 10^{7}$ and $2.90 \pm 0.0250 \times$ $10^{7} \Omega$.

\subsubsection{Methods for rapid analysis of EIS spectra}

Twenty different coated samples that had undergone a variety of exposure conditions, such as accelerated or outdoor weathering or immersion in electrolyte for 
various lengths of time were characterized using EIS. The protective quality of coatings was assessed by circuit modeling to categorize them into three groups: 1) those providing excellent protection (color coded green), 2) those providing adequate protection (color coded yellow), or 3) those that failed as a protective barrier (color coded red). Various models used to characterize coatings were shown in Figure 1.1 and the calculated impedance values of representative elements were compared in order to differentiate coatings' protective quality. The circuit elements selected for comparison were some of those associated with failure of coatings, and were either a resistor representative of the coating integrity: $R_{1}$ representing the pore resistance or $R_{2}$ representing the charge transfer resistance, or the largest capacitor in the system: $C P E_{3}$ ) which is related to exposed metal/oxide area. Using the value of the selected element, impedances $\left(Z_{R 1}, Z_{R 2}\right.$, or $\left.Z_{C P E 3}\right)$ were calculated and used to categorize the protective quality of each coated panel. Coatings having excellent protective properties had an impedance (of the selected element) greater than $10^{9} \Omega \cdot \mathrm{cm}^{2}$, adequate protective properties had impedances from $10^{7}-10^{8} \Omega \cdot \mathrm{cm}^{2}$, and poor protective properties had impedances less than $10^{6} \Omega \cdot \mathrm{cm}^{2}$. The data for twenty different coatings analyzed by this method are shown in Table 1.1, with the column heading 'Element Impedance'. To ascertain whether alternative methods of assessing protective quality could be used in place of circuit modeling and data fitting, other analysis methods that required less spectral interpretation were tested and compared against the classifications of the twenty samples determined by EEC modeling. Shown in Figure 1.6A-D are the numerical values produced by the alternative methods versus the EEC values (in impedance) used to categorize the protective quality of the coatings. 
Previously published methods to quickly analyze EIS data have included integrations of the Bode plot area ${ }^{23}$ and impedance ratios from various frequency ranges $^{18}$. Six of those methods were tested for validity using the twenty samples in this study and compared against the impedance values calculated from EEC modeling. None of the previously published methods produced easily identifiable categories or strong linear correlations; the data is presented and discussed in the appendix. This negative result necessitated further study to understand the relationship between the physical interpretation of the interface obtained by circuit modeling and the mathematical parameters extracted from impedance data.

Table 1.1 Values of parameters determined from EIS spectra used in the categorization of the protective quality of coatings for the EEC and slope methods. The frequency range used in each method is listed below the column heading. The protective quality determined by each method is given by: excellent (E), adequate (A), and poor (P). MPD: multiple point defects, soak: immersion in electrolyte.

\begin{tabular}{clccc}
\hline $\begin{array}{c}\text { Coating } \\
\text { Type }\end{array}$ & \multicolumn{1}{c}{ Weathering } & $\begin{array}{c}\text { Model/ } \\
\text { Element }\end{array}$ & $\begin{array}{c}\text { Element Impedance } \\
\left(\Omega \cdot \mathrm{cm}^{2}\right)\end{array}$ & Slope \\
& & $0.1-1 \times 10^{6} \mathrm{~Hz}$ & $0.1-1 \times 10^{6} \mathrm{~Hz}$ \\
\hline 1 & None & $1 / \mathrm{R}_{1}$ & $2.40 \times 10^{10}(\mathrm{E})$ & $-0.971(\mathrm{E})$ \\
1 & None & $2 \mathrm{~b} / \mathrm{R}_{1}$ & $3.29 \times 10^{7}(\mathrm{~A})$ & $-0.983(\mathrm{E}) \dagger$ \\
1 & Abrasion & $2 \mathrm{~b} / \mathrm{R}_{1}$ & $2.58 \times 10^{8}(\mathrm{~A})$ & $-0.972(\mathrm{E}) \dagger$ \\
1 & MPD & $3 / \mathrm{CPE}_{3}$ & $1.57 \times 10^{5}(\mathrm{P})$ & $-0.527(\mathrm{P})$ \\
1 & FL 9 mo & $3 \mathrm{~b} / \mathrm{CPE}_{3}$ & $7.95 \times 10^{6}(\mathrm{P})$ & $-0.929(\mathrm{E}) \dagger$ \\
1 & QUV-B 500 h & $2 \mathrm{~b} / \mathrm{R}_{1}$ & $2.78 \times 10^{8}(\mathrm{~A})$ & $-0.972(\mathrm{E}) \dagger$ \\
1 & QUV-B 500 h & $3 / \mathrm{CPE}_{3}$ & $2.36 \times 10^{6}(\mathrm{P})$ & $-0.758(\mathrm{P})$ \\
2 & PDX 15 mo & $2 / \mathrm{R}_{2}$ & $5.28 \times 10^{8}(\mathrm{~A})$ & $-0.945(\mathrm{E}) \dagger$ \\
2 & PDX 31 mo & $2 / \mathrm{R}_{2}$ & $2.02 \times 10^{7}(\mathrm{~A})$ & $-0.958(\mathrm{E}) \dagger$ \\
2 & QUV-B 500 $\mathrm{h}$ & $2 / \mathrm{R}_{2}$ & $5.18 \times 10^{8}(\mathrm{~A})$ & $-0.970(\mathrm{E}) \dagger$ \\
2 & QUV-B 1000 h & $3 \mathrm{~b} / \mathrm{CPE}_{3}$ & $1.52 \times 10^{7}(\mathrm{~A})$ & $-0.893(\mathrm{~A})$ \\
2 & QUV-B 1500 h & $3 \mathrm{~b} / \mathrm{CPE}_{3}$ & $8.64 \times 10^{6}(\mathrm{P})$ & $-0.715(\mathrm{P})$ \\
3 & None & $2 \mathrm{~b} / \mathrm{R}_{1}$ & $2.23 \times 10^{5}(\mathrm{P})$ & $-0.533(\mathrm{P})$ \\
3 & QUV-B 1250 h & $3 / \mathrm{R}_{2}$ & $7.24 \times 10^{5}(\mathrm{P})$ & $-0.818(\mathrm{P})$ \\
4 & 20 h soak & $1 / \mathrm{R}_{1}$ & $4.64 \times 10^{9}(\mathrm{E})$ & $-0.916(\mathrm{E})$ \\
4 & 68 h soak & $2 / \mathrm{R}_{2}$ & $1.86 \times 10^{8}(\mathrm{~A})$ & $-0.837(\mathrm{~A})$ \\
4 & 8 day soak & $3 / \mathrm{R}_{2}$ & $1.23 \times 10^{6}(\mathrm{P})$ & $-0.806(\mathrm{P})$ \\
5 & 25 h soak & $2 / \mathrm{R}_{2}$ & $1.36 \times 10^{10}(\mathrm{E})$ & $-0.951(\mathrm{E})$ \\
5 & 7 day soak & $2 / \mathrm{R}_{2}$ & $4.28 \times 10^{9}(\mathrm{E})$ & $-0.922(\mathrm{E})$ \\
5 & 62 day soak & $2 / \mathrm{R}_{2}$ & $4.23 \times 10^{9}(\mathrm{E})$ & $-0.889(\mathrm{~A}) \dagger$ \\
\hline
\end{tabular}

*multiple point defects, soak $=$ immersion in electrolyte, †denotes samples that were incorrectly categorized by that method. 
Table 1.2 Values of parameters determined from EIS spectra used in the categorization of the protective quality of coatings for the three variations of the estimated first derivative methods. The frequency range used in each method is listed below the column heading. The protective quality determined by each method is given by: excellent (E), adequate (A), and poor (P). MPD: multiple point defects, soak: immersion in electrolyte.

\begin{tabular}{|c|c|c|c|c|c|}
\hline $\begin{array}{c}\text { Coating } \\
\text { Type }\end{array}$ & Weathering & $\begin{array}{l}\text { Model/ } \\
\text { Element }\end{array}$ & $\begin{array}{c}\text { Minima } \\
\left(\mathrm{M} \Omega \cdot \mathrm{cm}^{2}\right)\end{array}$ & $\begin{array}{c}\text { Changing } \\
\text { Rate }|\mathrm{Z}| \\
\left(\mathrm{M} \Omega \cdot \mathrm{cm}^{2}\right)\end{array}$ & $\begin{array}{c}\text { Changing } \\
\text { Rate }|\mathrm{Z}| \\
\left(\mathrm{M} \Omega \cdot \mathrm{cm}^{2}\right)\end{array}$ \\
\hline & & & Variable & $0.1-1 \mathrm{~Hz}$ & $3 \mathrm{~Hz}$ \\
\hline 1 & None & $1 / \mathrm{R}_{1}$ & $-11,200(E)$ & $-5,020(\mathrm{E})$ & $-508(E)$ \\
\hline 1 & None & $2 \mathrm{~b} / \mathrm{R}_{1}$ & $-85.0(\mathrm{~A})$ & $-62.0(\mathrm{~A})$ & $-63.2(\mathrm{~A})$ \\
\hline 1 & Abrasion & $2 \mathrm{~b} / \mathrm{R}_{1}$ & $-969(\mathrm{~A})$ & $-455(\mathrm{~A})$ & $-111(\mathrm{~A})$ \\
\hline 1 & MPD* & $3 / \mathrm{CPE}_{3}$ & $-4.21(\mathrm{P})$ & $-4.04(\mathrm{P})$ & $-1.95(\mathrm{P})$ \\
\hline 1 & FL 9 mo & $3 \mathrm{~b} / \mathrm{CPE}_{3}$ & $-30.2(\mathrm{P})$ & $-16.1(\mathrm{P})$ & $-14.5(\mathrm{P})$ \\
\hline 1 & QUV-B $500 \mathrm{~h}$ & $2 \mathrm{~b} / \mathrm{R}_{1}$ & $-493(\mathrm{~A})$ & $-270(\mathrm{~A})$ & $-231(\mathrm{~A})$ \\
\hline 1 & QUV-B $500 \mathrm{~h}$ & $3 / \mathrm{CPE}_{3}$ & $-15.9(\mathrm{P})$ & $-10.1(\mathrm{P})$ & $-13.2(\mathrm{P})$ \\
\hline 2 & PDX 15 mo & $2 / \mathrm{R}_{2}$ & $-277(\mathrm{~A})$ & $-218(\mathrm{~A})$ & $-162(\mathrm{~A})$ \\
\hline 2 & PDX 31 mo & $2 / R_{2}$ & $-106(\mathrm{~A})$ & $-73.9(\mathrm{~A})$ & $-21.6(\mathrm{~A})$ \\
\hline 2 & QUV-B 500 h & $2 / \mathrm{R}_{2}$ & $-263(\mathrm{~A})$ & $-208(\mathrm{~A})$ & $-179(\mathrm{~A})$ \\
\hline 2 & QUV-B $1000 \mathrm{~h}$ & $3 \mathrm{~b} / \mathrm{CPE}_{3}$ & $-162(\mathrm{~A})$ & $-107(\mathrm{~A})$ & $-59.1(\mathrm{~A})$ \\
\hline 2 & QUV-B $1500 \mathrm{~h}$ & $3 \mathrm{~b} / \mathrm{CPE}_{3}$ & $-20.0(P)$ & $-15.9(\mathrm{P})$ & $-12.9(\mathrm{P})$ \\
\hline 3 & None & $2 \mathrm{~b} / \mathrm{R}_{1}$ & $-4.41(\mathrm{P})$ & $-2.51(\mathrm{P})$ & $-0.757(\mathrm{P})$ \\
\hline 3 & QUV-B $1250 \mathrm{~h}$ & $3 / \mathrm{R}_{2}$ & $-5.40(\mathrm{P})$ & $-1.78(\mathrm{P})$ & $-4.25(\mathrm{P})$ \\
\hline 4 & 20 h soak & $1 / R_{1}$ & $-3,920(E)$ & $-3,100(E)$ & $-892(\mathrm{E})$ \\
\hline 4 & 68 h soak & $2 / \mathrm{R}_{2}$ & $-89.4(\mathrm{~A})$ & $-33.4(\mathrm{~A})$ & $-89.4(\mathrm{~A})$ \\
\hline 4 & 8 day soak & $3 / \mathrm{R}_{2}$ & $-23.9(\mathrm{P})$ & $-3.29(\mathrm{P})$ & $-16.6(P)$ \\
\hline 5 & 25 h soak & $2 / R_{2}$ & $-4,750(E)$ & $-2,990(E)$ & $-802(\mathrm{E})$ \\
\hline 5 & 7 day soak & $2 / R_{2}$ & $-2,180(\mathrm{E})$ & $-1,570(E)$ & $-548(E)$ \\
\hline 5 & 62 day soak & $2 / \mathrm{R}_{2}$ & $-3,080(\mathrm{E})$ & $-1,490(E)$ & $-475(E)$ \\
\hline
\end{tabular}

*multiple point defects, soak $=$ immersion in electrolyte, $\dagger$ denotes samples that were incorrectly categorized by that method. 


\subsubsection{Slope}

The capacitive element of an ideal protective coating should have a slope that is close to -1 in a Bode plot and capacitance should dominate most of the normal frequency range $(0.1 \mathrm{~Hz}$ and $100 \mathrm{MHz})$. Two data points from the middle part of that range $(1 \mathrm{kHz}$ and $100 \mathrm{kHz})$ were selected, the slope calculated, and the quality of the coating was assessed by the deviation from the ideal for various weathered and intentionally damaged coatings, as can be seen in Table 1.1. From those data, categories of protective quality were delineated here as follows: green/excellent for slopes between -1.00 and -0.900 , yellow/adequate for slopes between -0.899 and -0.820 , and red/failed for slopes greater than -0.820 . A plot of protective quality by slope versus EEC elements was produced to compare those two categorization methods, as can be seen in Figure 1.6A.

When comparing EEC categorization and the slope method in Figure 1.6A, several samples fell outside of the boundaries as determined above; alternative category boundaries also produced outlying data points. The correlation coefficient $\left(\mathrm{R}^{2}\right)$ was unacceptably low at 0.548 . The presence of outliers suggested that this determination was not an acceptable alternative method for assessing protective quality. (Different selected frequencies used to determine the slope did not produce an acceptable result either.) A review of the EIS spectra from these panels showed that two different spectral characteristics caused coatings to fall outside of the boxed boundaries: 1) the presence of two time constants and 2) a defect in the film. When two time constants appear within the same range that was used to determine the slope the deviation in linearity of the Bode plot is not discerned. That situation was observed, for example, in a Tnemec 
coated panel immersed in electrolyte for 62 days which showed two time constants, likely caused by the presence of two different layers within the coating: an intact portion of the coating which was observed at high frequencies and a second electrolytesaturated layer of the coating observed at mid-range frequencies. Defects in coatings may or may not be discernable by the slope of the impedance versus frequency because the capacitance of intact coatings will dominate at mid-high range frequencies (above approximately $100 \mathrm{~Hz}$ ), while a defect (if present) will be observed as a resistor dominant at lower frequencies and reduce the value of the total impedance. In six cases, the presence of a defect in the coating led to a decrease in the total impedance, but that occurred at frequencies lower than the frequency range used to calculate the slope. These outliers can be seen in Figure 1.6A as data points lying outside of the shaded boxes. The misclassification of those coated panels warranted the exploration of other quicker analysis methods that may have greater accuracy.

\subsubsection{First derivative approximations from Impedance modulus plots}

A second method tested for rapidly analyzing EIS data was by plotting an approximation of the first derivative by parabolic fit, following Equation 1.3:

$$
k^{\prime}(f)=\frac{d(|Z|)}{d(\log (f))}
$$

where $|\mathrm{Z}|$ is the impedance at the respective frequencies $(f)$. Equation 1.3 was used in three different ways: 1) plot of the first derivative at all measured frequencies, 2) an approximation of the first derivative at two log averaged frequencies $(0.1$ and $1 \mathrm{~Hz})$ and

3) the derivative at a single frequency $(3 \mathrm{~Hz})$. 
The full first derivative of the impedance modulus plotted against the measured frequencies for all twenty coatings are shown in Figure 1.7. In those plots, the minimum of each trace is indicative of the coating condition. The categories of coatings were separated and plotted in Figures 1.7B-D. The minima from these plots provided values listed in Table 1.2 and categories of protective quality were delineated: excellent protection was defined here when the minimum is less than $-1.5 \mathrm{G} \Omega \cdot \mathrm{cm}^{2}$ (plotted in Fig. 1.7B), coatings providing adequate protection was defined when the minimum is between $-1.5 \mathrm{G} \Omega \cdot \mathrm{cm}^{2}$ and $-60.0 \mathrm{M} \Omega \cdot \mathrm{cm}^{2}$ (plotted in Figure $1.7 \mathrm{C}$ ), while little protection against corrosion was defined when the minimum is greater than -60.0 $\mathrm{M} \Omega \cdot \mathrm{cm}^{2}$ (plotted in Fig. 1.7D). The correlation coefficient in Figure 1.6B was 0.935, which is the highest value by any of the methods. While providing a perfect match
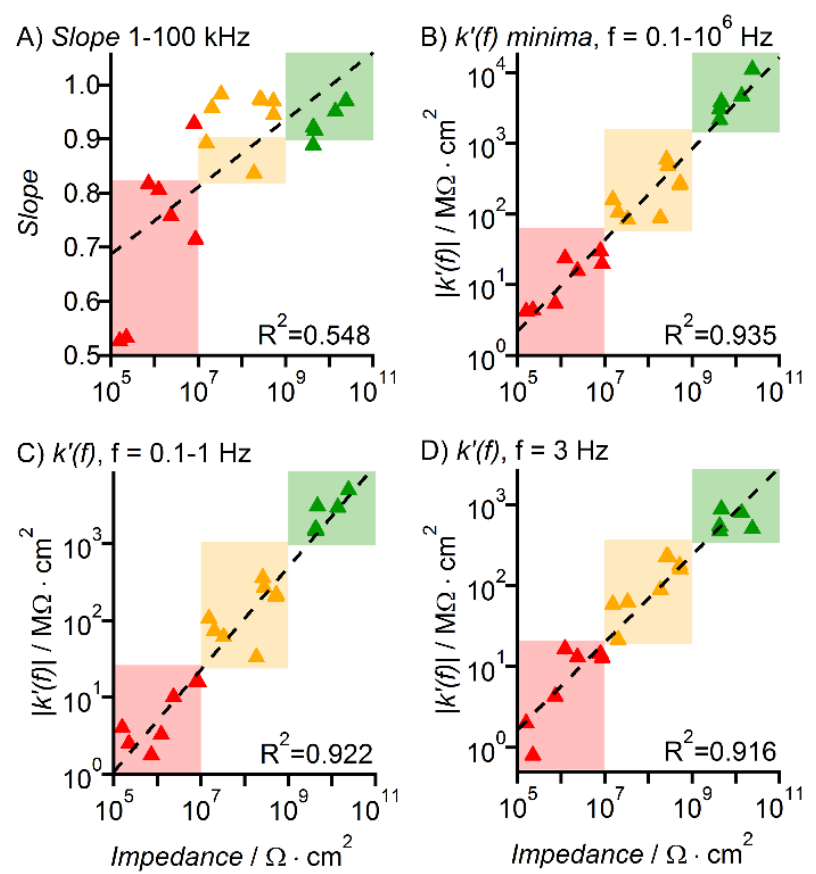

Figure 1.6 Correlation plots of classification scheme determined by: A) the slope between 1 and 100 $\left.\mathrm{kHz}, \mathrm{y}=0.0278 \ln (\mathrm{x})+0.3603 ; \mathrm{R}^{2}=0.5476 ; \mathrm{B}\right)$ the minimum value obtained from the $\mathrm{k}^{\prime}(\mathrm{f})$ approximation, $\mathrm{y}=0.0013 \mathrm{x}^{0.646} ; \mathrm{R}^{2}=0.9349 ; \mathrm{C}$ ) an approximation of the first derivative between 0.1 and $1.0 \mathrm{~Hz}, \mathrm{y}=0.0005 \mathrm{x}^{0.6661} ; \mathrm{R}^{2}=0.9215$; and $\left.\mathrm{D}\right)$ the approximation of the derivative at $3 \mathrm{~Hz}, \mathrm{y}=$ $0.0033 \mathrm{x}^{0.5401} ; \mathrm{R}^{2}=0.9157$ versus the EEC classification scheme. 
between the EEC model classification scheme and a high $\mathrm{R}^{2}$ value, this method required acquisition of large range of the EIS spectrum in the analysis because the minimum of the first derivative approximation occurred at different frequencies (ranging from 0.1 to $20 \mathrm{~Hz}$ ) for each coating.

We then investigated whether it was necessary to acquire an impedance spectrum over two orders of magnitude of frequency (or more such as in the case of the minimum of the first derivative described above) or whether data from a narrower range (e.g. one order of magnitude or a single data point) could be used to accurately categorize the protective quality of coatings. Because additional circuit elements (such as the capacitance of an oxide layer, which forms and is seen by EIS in coatings that are failing) influence the lower frequency portion of the spectrum, the range chosen at such lower frequencies were 0.1 and $1 \mathrm{~Hz}$, according to Equation 1.4.

$$
k^{\prime}(f)=\frac{\left|Z_{1}\right|-\left|Z_{0}\right|}{\log \left(f_{1}\right)-\log \left(f_{2}\right)}=\frac{\Delta|Z|}{\Delta \log (f)}
$$

The difference in impedance derivative from 0.1 to $1 \mathrm{~Hz}$ provided values listed in Table 1.2 and corresponding category assignments are as follows: excellent protective qualities can be offered when the value is less than $-1 \mathrm{G} \Omega \cdot \mathrm{cm}^{2}$, adequate protection is offered when the value is between $-1 \mathrm{G} \Omega \cdot \mathrm{cm}^{2}$ and $-25.0 \mathrm{M} \Omega \cdot \mathrm{cm}^{2}$, and little protection is offered when the value is greater than $-25 \mathrm{M} \Omega \cdot \mathrm{cm}^{2}$. Comparing this method of classifying coatings to the EEC modeling method also gave a perfect category correlation for all twenty samples and a good $\mathrm{R}^{2}$ value of 0.922 , as can be seen in Figure 1.6C. 
Because the minimum of the first derivative approximation occurred at different frequencies for each coating, it is important to note that selecting a single frequency that accurately represents the overall protective quality of the coating is potentially problematic. Despite that potential pitfall, we investigated whether it is possible to utilize a single impedance data point at a key frequency to categorize the protective quality of coatings using Equation 1.3 at $3 \mathrm{~Hz}$. That frequency was selected because, from our data, the influence of additional circuit elements is most often observable at that approximate frequency. The first derivative approximation at $3 \mathrm{~Hz}$ provided values listed in Table 1.2 and from these data, categories of protective quality were delineated.

A)

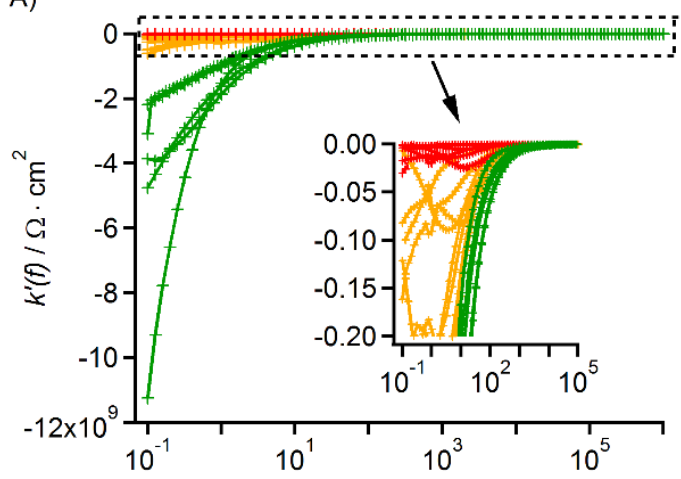

C)

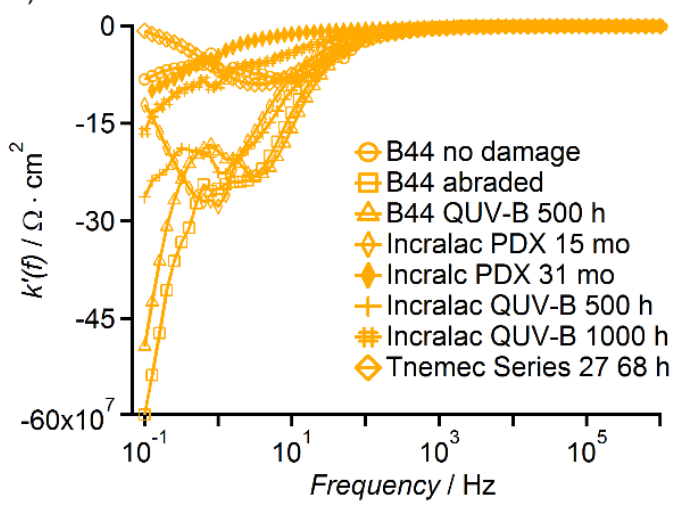

B)

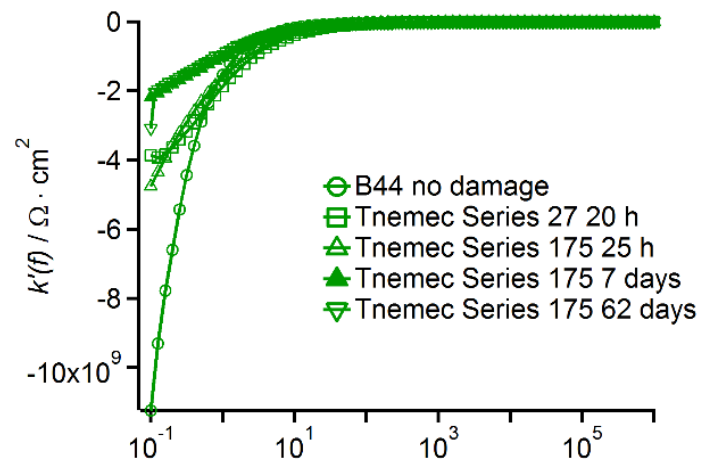

D)

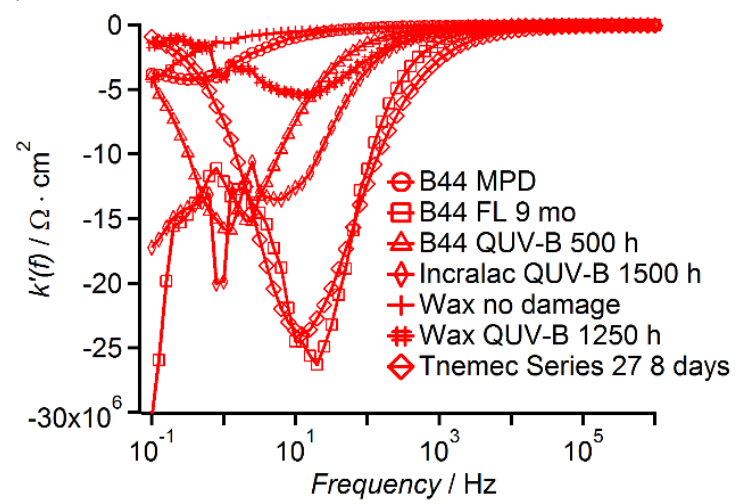

Figure 1.7 An approximation of the first derivative by parabolic fitting of EIS data from coated metal panels: all spectra are shown in A) with a magnified view shown in the inset and separated by protective quality: B) excellent, C) adequate, or D) poor protection in the remaining plots. 
Excellent protective qualities are defined here as having values less than $-350 \mathrm{M} \Omega \cdot \mathrm{cm}^{2}$, adequate protection is defined when the values are between $-350 \mathrm{M} \Omega \cdot \mathrm{cm}^{2}$ and -20.0 $\mathrm{M} \Omega \cdot \mathrm{cm}^{2}$, while those offering poor protection had values above $-20.0 \mathrm{M} \Omega \cdot \mathrm{cm}^{2}$. This method also gave a perfect category correlation to the EEC categorizations as seen in Figure 1.6D.

\subsection{Conclusions}

Coatings having intentional defects were compared to weathered coatings and similarities in their EIS spectra were found. Two different EEC models best represented the two different types of intentional defects and each of those models could also represent weathered coatings. Intentional abrasion of coated panels showed EIS spectral features that were similar to coated panels weathered for 500 hours in a QUV-B chamber, while a coating with multiple pinhole defects was spectrally similar to an imperfectly applied coating that was weathered for 500 hours in a QUV-B chamber.

Electrochemical signatures observed from these data can aid in the characterization of coatings, particularly when an EIS spectral frequency range is one or more orders of magnitude. Even so, perfect category correlation was found when only a single frequency was used $(3 \mathrm{~Hz})$. From this work we showed that it is possible to categorize the protective quality of coatings using simpler and faster methods of data analysis than EEC modeling. Perfect correspondence to the categories obtained from EEC modeling was obtained in three of the four cases: 1) when using the minima from the full EIS spectra, 2) the approximation of the first derivative for a range between 0.1 and $1 \mathrm{~Hz}$ and 3) the first derivative approximation of a single point at $3 \mathrm{~Hz}$. Use of the 
slope of the impedance modulus over the frequency range $1 \mathrm{kHz}-100 \mathrm{kHz}$ correlated with the EEC modeling in twelve of the twenty coatings tested.

The boundaries delineated by each method are based on the observations of these twenty coatings of five different coating types. Their actual values may vary somewhat as additional/different data points are acquired. The primary purpose in determining categories is not intended to provide specific numerical values for boundaries, but rather to show that the data clusters and shows a linear correlation in these plots. In fact, depending on the specific application being investigated, it may be advantageous to shift the boundary cut-offs to affect the sensitivity of the category determination or to create additional categories aside from the three shown here.

Accurate categorization of the protective quality of coatings is one of the parameters in selecting which data analysis method is most appropriate to use; additional considerations are the selected frequency range and the impedance limits of different spectrometers. For example, the slope of the impedance which was taken at frequencies in the $\mathrm{kHz}$ range enables fast data acquisition (with a spectrum from $1 \mathrm{kHz}$ - $100 \mathrm{kHz}$ taking fractions of a second to complete) or the first derivative approximation centered at $3 \mathrm{~Hz}$ may take a few seconds to acquire. While measurements that span a larger frequency range may be more likely to reveal deviations from ideally protective qualities of coatings (depending on the range), acquiring those data sets is more time consuming ${ }^{26}$. For example, taking an EIS measurement between 0.01 and $1 \mathrm{MHz}$ may take approximately 30 minutes, while an EIS measurement from 0.1 to $1 \mathrm{MHz}$ may take only 5 minutes, depending on the instrument. Impedances of the spectra increase as the frequency of the measurement 
decreases, and the total impedance of the system may exceed that of the instrument, particularly at lower frequencies, making measurements impossible. Another important consideration in both selecting frequency ranges for measurement (and evaluating data from those ranges) is noise and the possible need for data smoothing, particularly when taking measurements in the field which may have sources of noise at frequencies that interfere with data analysis. The first derivative approximation by parabolic fit that was applied in two of the methods tested here had the effect of data smoothing that may contribute to more accurate data analysis from field-acquired data.

In this work we used circuit modeling to provide a physical interpretation of the systems under study, reported physical parameters of circuit elements and applied simple mathematical interpretations of the impedance data (e.g. first derivative approximations), to distinguish the protective quality of different coatings. In some of the linear correlation plots (as in Fig 6B-D) we showed that the protective quality predictions from the mathematical and physical models are the same. It has been suggested by others, that future impedance studies aimed at quicker analysis may include artificial neural networks (ANN), which may include principle component analysis 20-22, 27-28; some of the data presented here supports the validity of those suggested approaches for evaluating the protective quality of coatings electrochemically as demonstrated by the three plots having correlation coefficients exceeding 0.9. However, highlighted by the seven plots (Fig 6A and Fig A.1) that show poor correlation (having coefficients ranging between 0.69 and 0.38 ), a mathematicalonly determination may fail to correctly ascertain protective quality. This conclusion suggests that it is important to first thoroughly characterize interfaces by circuit 
modeling and then such categories determined by that physical interpretation may be used to appropriately train the ANN or other mathematical interpretation algorithm. 


\section{Understanding lateral pathway measurements by co-planar hydrogel electrochemical cells for characterizing organic layers on surfaces}

Assessing coating quality on sculptures in the field using a standard electrochemical cell for electrochemical impedance spectroscopy (EIS) measurements is problematic in its destructive nature. A portion of coating must be removed and only planar substrates can be measured with the commonly used cell. To ensure the measurement is nondestructive and flexible to any surface shape, a co-planar hydrogel electrochemical cell was explored for EIS measurements on multiple organic surfaces. The similarities and differences between the standard electrochemical cell and co-planar hydrogel cells were studied through multiple experiments and applied to assess the quality of protective organic coatings on a coated steel sculpture in the field. Both cells were able to measure coating capacitance which can give information about bulk coating degradation. Sheet resistance was measured only when the co-planar hydrogel cell was used and was indicative of an even earlier warning sign of degradation of the surface of the protective coating.

\subsection{Introduction}

Corrosion is a spontaneous, largely irreversible process that occurs on metals with significant economic impacts. For example, the National Association of Corrosion Engineers estimated that in 1998, 3.1\% of the Gross National Product (GNP) or \$276 billion, was the direct cost of corrosion ${ }^{29}$. Given the high costs of corrosion, efforts such as application of corrosion inhibitors and protective coatings are made to prevent corrosion. However, those methods fail over time as organic molecular components within those formulations are subject to the usual array of oxidizers such as UV light 
or through thermal mechanisms such as temperature cycling with its accompanying mechanical changes that occur in an outdoor environment ${ }^{30}$. In addition, the protective period of such anti-corrosion material is fairly short ${ }^{31}$. It would be advantageous to have the ability to measure coating degradation before coatings fail to provide adequate protection against corrosion. A non-destructive early warning system for field use to detect coating failure before damage to the underlying substrate has occurred does not currently exist. Such an instrument would have applications in metal infrastructure, such as buildings and bridges and is of particular importance to the field of material cultural heritage, specifically outdoor metal sculptures ${ }^{32}$. Electrochemial impedance spectroscopy (EIS) is a widely used technique to determine the electrochemical properites of a variety of materials including semiconductors, electrochemical power sources, corrosion, and protective coatings ${ }^{33}$. The traditional way of measuring impedance includes a three electrode cell immersed in an electrolyte solution. The applied AC potential is perpendicular to the surface of the material being measured and therefore provides information about the bulk of the material such as double-layer capacitance, charge transfer resistance, material capacitance and resisitance, and diffusion properties. EIS is frequently used to assess coating condition for use on metal substrates, including sculptures ${ }^{34-35}$; however, the technique possesses significant limitations that prevent its use in the field. The common method utilizes a rigid glass, liquid electrolyte-filled fluid cell and direct electrical contact to the metal substrate (requiring removal of a portion of the coating to make the measurement). Given the geometric requirements and the destructive nature of coating removal, the technique is not feasible for many relevant outdoor substrates, such as sculptures, buildings, bridges, 
airplanes and other valuable structures that have non-planar and non-horizontal portions. However, on planar, horizontal test panels, the traditional EIS setup using rigid glass fluid cells does provide significant insights into the degradation of coatings ${ }^{36-39}$.

Alternative geometries to the standard fluid cell have been previously explored for the purpose of making measurements in situ, yet none address the requirement of making measurements non-destructively. For example, in three studies the substrate must be exposed by removing some of the protective coating ${ }^{40-42}$, and in two other studies, damaged coatings must be pre-soaked with electrolyte to enable the measurement, causing potential damage to the substrate ${ }^{43-44}$. In the latter two studies, without pre-treatment, the electrode setups were only able to provide information about the surface of the protective coating because their soft-polymer electrodes did not have electrolyte to penetrate micropores in order to obtain diffusion and charge transfer information. Even with pre-treatment, the cells were only able to provide comparable data to the standard liquid cell at high frequencies. In previous work out of our lab, we have developed a method using surface-mounted flexible electrodes with novel hydrogels as the solid electrolyte for EIS data collection that enables measurements to be made on non-planar substrates and without removal of the coating ${ }^{45-46}$. Here, using the co-planar hydrogel cell geometry, we thoroughly investigate the different impedance responses at different frequencies and present data demonstrating that the coating capacitance and other circuit elements dominate the measurement of some systems, while for very high impedance systems, the EIS measurement is largely a measure of the sheet resistance of the coating. Sheet resistance can be used to monitor 
the surface degradation of the coating. The responses of each system provides useful benchmarks for evaluating the protective quality of different coatings in situ.

\subsection{Experimental}

\subsubsection{Materials}

Soda lime, Pilkington Optiwhite ${ }^{\mathrm{TM}}$, and NSG TEC ${ }^{\mathrm{TM}} 250$ glass was obtained from Pilkington and cut into $\sim 2 \times 4$ in rectangles. TEC ${ }^{\mathrm{TM}} 250$ glass had a conductive coating on its surface with a reported sheet resistance of 260-325 $\Omega / \square$. Carboxyethylsilanetriol (CTES) disodium salt (25\% in water) and hexadecyltrimethoxysilane (HDTMS) (95\%) were purchased from Gelest. Silver foil $(99.99 \%, 0.050 \mathrm{~mm}$ thickness) was purchased from Advent Research Materials. Coatings used in this study were Tnemec Series 27F.C. Typoxy® and Tnemec Series 175-Endura-Shield® topcoat with N69-Hi-Build Epoxoline® II primer. The experimental panels were pre-painted by Tnemec on $8 \mathrm{~cm}$ $\times 15 \mathrm{~cm}$ steel.

\subsubsection{Electrochemical Impedance Spectroscopy}

EIS measurements were obtained using a Gamry Reference 600 Potentiostat with Gamry Framework 6 software. EIS data was acquired from $1 \mathrm{MHz}$ to $0.1 \mathrm{~Hz}$ or $0.01 \mathrm{~Hz}$ (10 points per logarithmic decade). Co-planar hydrogel cells consisted of either two $18 \mathrm{~cm}^{2}$ (protective coating measurements) or $6 \mathrm{~cm}^{2}$ (functionalized glass measurements) hydrogels with silver foil as the working and counter electrodes. Exact gel area was measured using calipers and assigned a $10 \%$ error. The distance between working and counter electrodes was $4 \mathrm{~mm}$ for protective coating measurements and 10 $\mathrm{mm}$ for functionalized glass measurements. An $\mathrm{AC}$ potential of $60 \mathrm{mV}_{\mathrm{rms}}$ and a $\mathrm{DC}$ 
potential of $0.0 \mathrm{~V}$ vs open circuit potential (which ranged between 200 and $-200 \mathrm{mV}$ ) was applied. Three spectra were acquired for each measurement to monitor stability of the system. Spectra were normalized using the $\mathrm{K}_{\text {cell }}$ constant found in our previous work $^{45}$. EIS Spectra were analyzed using EEC models constructed using Gamry Echem Analyst and fit using the Simplex method. CPEs were commonly used in the models in place of capacitors to better describe the non-ideal nature of the system. Impedance of individual circuit elements were calculated for resistors:

$$
Z_{R}=R
$$

where $R$ is $\Omega \cdot \mathrm{cm}^{2}$ and for constant phase elements:

$$
Z_{C P E}=1 / Y_{0}(j \omega)^{\alpha}
$$

where $Y_{0}$ is $\mathrm{nS} \cdot \mathrm{s}^{\alpha} \cdot \mathrm{cm}^{-2}, j$ is $(-1)^{1 / 2}, \omega$ is the angular frequency, and $\alpha$ is $0 \leq \alpha \leq 1$ (an $\alpha$ of 0 is a pure resistor and an $\alpha$ of 1 is a pure capacitor).

\subsubsection{SEM}

SEM images were acquired with an FEI Sirion XL30 FEG SEM. An accelerating voltage of $3 \mathrm{keV}$ and working distance of 4.8-4.9 mm was used. Samples were gold coated using a PELCO 91000 Sputter Coater.

\subsubsection{Modification of glass substrates with silanes}

\subsubsection{Glass cleaning procedure}

Glass was soaked in a base bath overnight. The glass was then immersed in a 1:1 solution of $\mathrm{HCl}: \mathrm{MeOH}$ for 30 minutes. Piranha $\left(3: 1 \mathrm{H}_{2} \mathrm{SO}_{4}: 30 \% \mathrm{H}_{2} \mathrm{O}_{2}\right)$ ) was then 
used to etch the glass for 30 minutes. A final rinse was done with DI water. Glass was dried with nitrogen between each step and before silanization.

\subsubsection{Functionalization with silanes}

Freshly cleaned glass was immersed for one hour in a solution of $0.5 \%$ HDTMS or CTES in methanol by weight. HDTMS containers were purged with nitrogen before sealing to encourage a dry environment during functionalization. The glass was then rinsed with methanol and sonicated for 30 minutes. Substrates were then heated at 110 ${ }^{\circ} \mathrm{C}$ for 20 minutes. EIS measurements were taken when substrates reached room temperature.

\subsection{Results and Discussion}

\subsubsection{Sheet resistance measurements on glass surfaces}

\subsubsection{Sheet resistance measurements of a glass having a known sheet resistance}

The co-planar hydrogel cell was mounted onto a commercially available coated glass (TEC $\left.{ }^{\mathrm{TM}} 250\right)$ and measured by EIS using three different hydrogel widths, constant length, and constant spacing. The width and spacing are illustrated in Figure 2.1A. The raw EIS of TEC ${ }^{\mathrm{TM}} 250$ glass the varying cell configurations is shown in Figure 2.1B. As seen in the inset, the circuit model fit to the raw data consisted of a CPE that changed with varied gel width in series with two resistors and a CPE in parallel that did not change with gel width. The $\mathrm{CPE}$ element that dominated from mid-low range frequences which varied with gel width was attributed to the capacitance of the coating on the glass. As the area of the gels changed as the gel width was decreased, the CPE value changed by the same ratio - confirming the mutual dependence of gel area and 
measured capacitance. The resistor that dominated from $10^{4}$ to $10^{6} \mathrm{~Hz}$, which did not change with gel area was attributed to the sheet resistance of the glass. When normalized to the geometry of the co-planar hydrogel cell (accouting for length of and spacing between the gels) and assuming these parameters hold to the sheet resistance relationship, this value was $303 \pm 9.22 \Omega / \square$. Note that this value closely compares to the reported sheet resistance of $260-325 \Omega / \square$ for this glass. More typically, sheet resistance measurements are taken using a four point probe on a dry conductive substrate. In the co-planar hydrogel gel setup described in this work, because there is electrolyte present within the hydrogels, electrolyte can premeate into the coating of the glass. Indeed, that phenomenon was observed in the inflection between $10^{3}$ and $10^{4} \mathrm{~Hz}$ which did not change with gel width. The slightly greater resistive element $(837 \pm 23.3$ $\Omega / \square$ after normalization) and CPE element that had an alpha value close to that of a diffusion element ( $\sim 0.2$ vs 0.5 respectively) suggested that there exists a pathway through the coating that permeates more deeply than simply electrolyte wicking across the surface. This data confirms that sheet resistance is the measured parameter and demonstrates that this measurement techinque makes additional information about the material available, such as coating capacitance and diffusive properites with the use of the co-planar hydrogel electrochemical hydrogel cell. This technique is also valuable in that the hydrogels are conformable to non-planar, non-lateral substrates and because the size of the hydrogel is easily variable, measurements can be made on small or large areas (ranging from a few millimeters to tens of centimeters). 
A)

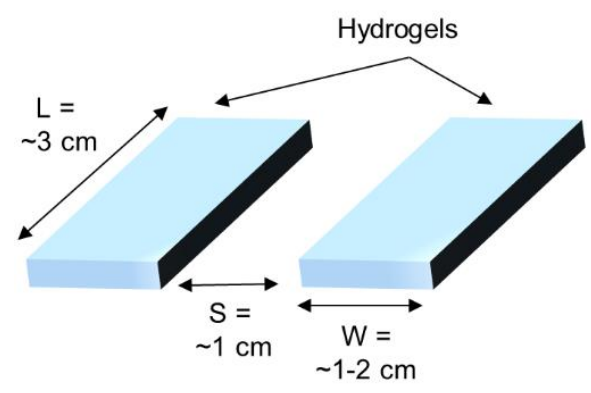

B)

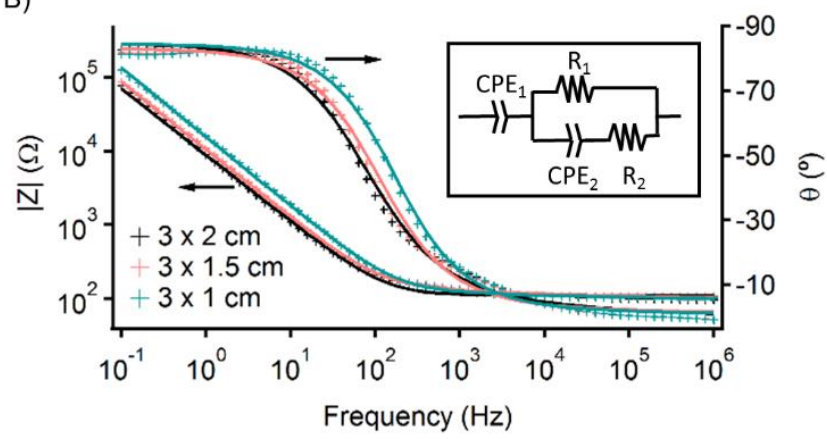

Figure 2.1 A) An illustration of the co-planar hydrogels with the fixed length, fixed spacing, and variable width labeled as shown. B) Raw EIS of TEC ${ }^{\mathrm{TM}} 250$ glass with the modeled EEC inset. Model fits are displayed as solid traces. Total gel area for the approx. $3 \times 2 \mathrm{~cm}$ gels was measured after compression to be $10.72 \mathrm{~cm}^{2}$. The model fit contained the following circuit elements and their respective values: $\mathrm{CPE}_{1}: 2.01 \pm 0.0175 \times 10^{-5}, \alpha: 0.940 \pm 0.00233, \mathrm{R}_{1}: 347 \pm 4.97, \mathrm{CPE}_{2}: 9.57 \pm$ $0.834 \times 10^{-4}, \alpha: 0.310 \pm 0.00986, \mathrm{R}_{2:}: 126 \pm 1.11$. Total gel area for the approx. $3 \times 1.5 \mathrm{~cm}$ gels was 9.418 $\mathrm{cm}^{2}$. The values of the circuit elements for the model fit were: $\mathrm{CPE}_{1}: 1.75 \pm 0.0211 \times 10^{-5}, \alpha: 0.922 \pm$ $0.00305, \mathrm{R}_{1}: 347 \pm 5.68, \mathrm{CPE}_{2}: 2.80 \pm 0.262 \times 10^{-3}, \alpha: 0.181 \pm 0.00933, \mathrm{R}_{2}: 126 \pm 1.38$. Total gel area for the approx. $3 \times 1 \mathrm{~cm}$ gels was $6.458 \mathrm{~cm}^{2}$. The values of the circuit elements for the model fit were: $\mathrm{CPE}_{1}: 1.15 \pm 0.00949 \times 10^{-5}, \alpha: 0.938 \pm 0.00201, \mathrm{R}_{1}: 350 \pm 5.83, \mathrm{CPE}_{2}: 3.31 \pm 0.348 \times 10^{-3}, \alpha: 0.194 \pm$ $0.0105, \mathrm{R}_{2}: 127 \pm 1.09$.

\subsubsection{Varying co-planar hydrogel cell geometry}

To demonstrate the flexible geometry of the hydrogels, the geometry of the coplanar hydrogel cell was varied on functionalized soda lime glass. By varying the spacing and width, it was also possible to determine whether sheet resistance could be measured on such a high impedance material. Because soda lime glass is not a wellcontrolled surface due to strained surface bonds, carboxyethylsilanetriol (CTES) was used to produce a more controlled surface chemistry. EIS spectra were fit using the equivalent circuit model shown in the inset of Figure 2.3A. A resistive element was present from mid-low range frequencies. The pathway having the highest resistance in this co-planer cell is that of electrolyte ions traveling laterally through the silane monolayer surface of the glass. The capacitive portion of the plot is assigned to the 
coupling capacitance of the silver foil electrodes of the cell that are stationary and parallel to each other.

The magnitude of applied AC potential was also varied to confirm that there was no effect on impedance under larger applied potentials. The value of the resistive element in the EEC model fit was used to compare the relationship of sheet resistance to varied cell geometry. Gel spacing showed a direct linear dependence on resistance as seen in Figure 2.2A. Varying width of the hydrogels (Fig. 2.2B) showed that the resistive element is independent of width. Likewise, the magnitude of the AC potential (Fig. 2.2C) did not affect the measured resistance and demonstrated that a higher applied potential, up to $60 \mathrm{mV}$, was non-destructive for this measurement. Based on the linear dependence of the resistive element with gel spacing and the absence of resistance change associated with gel width, we conclude that the impedance change is a measurement of the sheet resistance of the silane layer. This confirms that sheet resistance of relatively high impedance materials can be measured using the co-planer EIS cell.
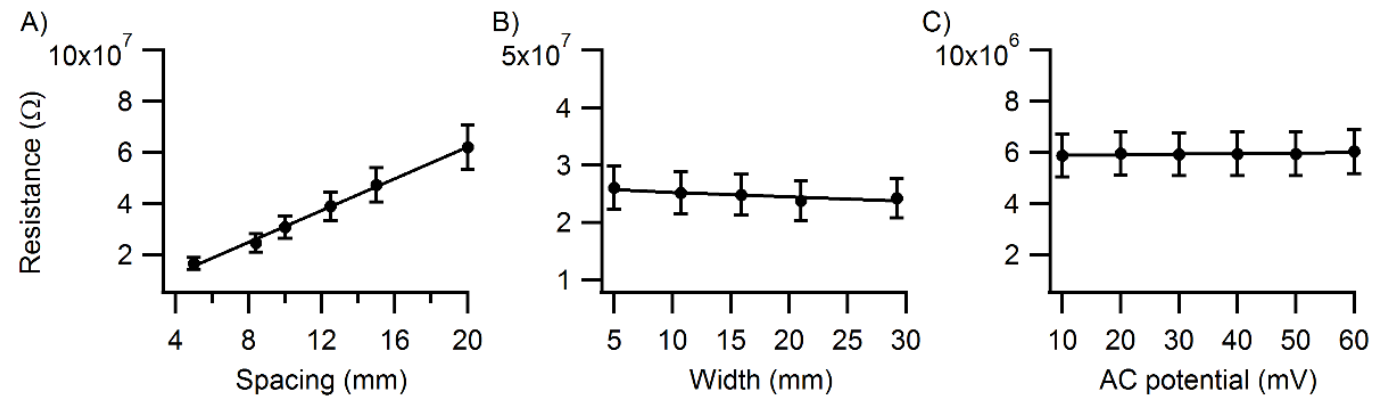

Figure 2.2 The resistance from the model fit for A) various spacing between hydrogels: $y=3.112 \times 10^{6} \mathrm{x}$ $-5.616 \times 10^{4}, R=0.9973, B$ ) various width of gels: $y=-8.125 \times 10^{4} x+2.616 \times 10^{7}$, and C) various applied AC potentials: $\mathrm{y}=2.323 \times 10^{3} \mathrm{x}+5.868 \times 10^{6}$. 


\subsubsection{EIS sheet resistance measurements sensitive to monolayer surface changes}

Soda lime and Optiwhite ${ }^{\mathrm{TM}}$ (modified soda lime) glass were functionalized using CTES and HDTMS to investigate the sensitivity of sheet resistance measurements to differences in surface monolayer chemistry on this relatively high impedance material. EIS was performed on the surfaces of the glass using the co-planar hydrogel cell. The raw EIS spectra were fit to the EEC model shown in the inset of Figure 2.3A. The resistive element of the model fit was then normalized to the length of and space between hydrogels to obtain sheet resistance. The capacitive element of the fit (from approx $10^{6}-10 \mathrm{~Hz}$ ) was left un-normalized and was assigned to the capacitive coupling between silver foil leads which did not change in value throughout measurements. These normalized model fit traces can be seen in Figure 2.3A. Calculated sheet resistances are displayed in Figure 2.3B. When functionalized with CTES, two different types of glasses (soda lime and Optiwhite ${ }^{\mathrm{TM}}$ ) had comparable sheet resistances of 189 $\pm 13.5 \mathrm{M} \Omega / \square$ and $174 \pm 45.1 \mathrm{M} \Omega / \square$, respectively. Similarly, when functionalized with a different silane, HDTMS, the two different glass types had comparable sheet resistances of $4.25 \pm 2.74 \mathrm{G} \Omega / \square$ and $2.38 \pm 1.00 \mathrm{G} \Omega / \square$, respectively, which were greater in value than when functionalized with CTES. The difference in sheet resistances between the two surface termination chemistries is likely due to the difference in hydrophilicity of the silanized glasses; HDTMS-modified glass, being more hydrophobic and a higher value, while CTES, being more hydrophilic has a smaller sheet resistance value. 

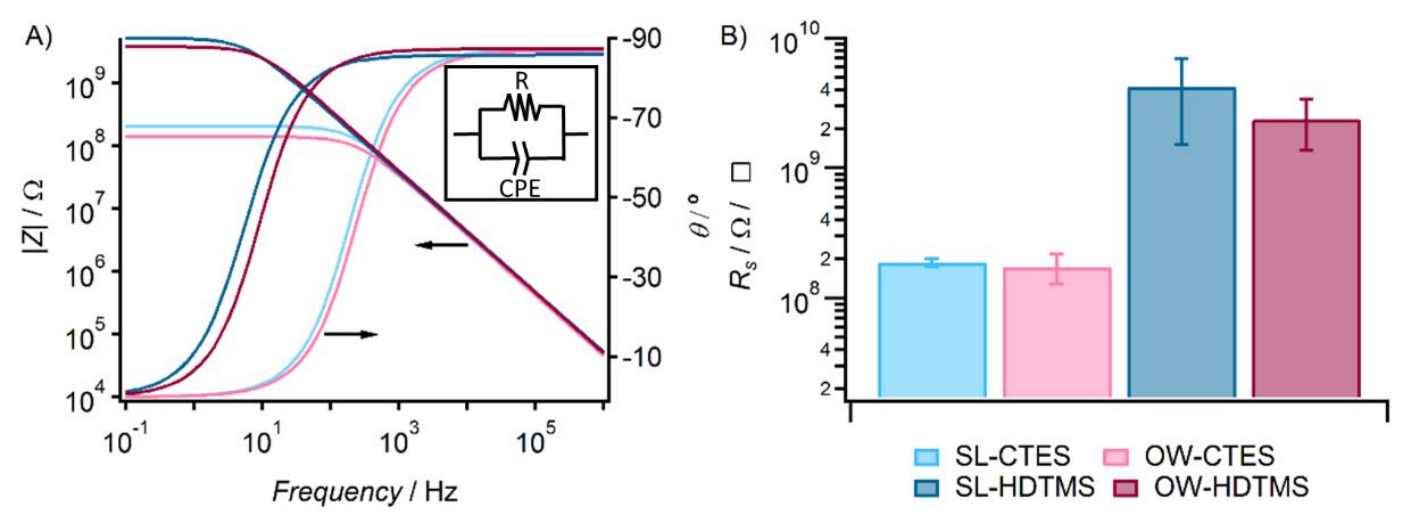

Figure 2.3 A) Normalized model fits for CTES and HDTMS glass surfaces. B) Sheet resistances of functionalized soda lime and Optiwhite ${ }^{\mathrm{TM}}$ glass.

\subsubsection{In-lab sheet resistance measurements on painted test panels}

To more fully vet the co-planar hydrogel cell before using in the field (i.e. on artwork), it is important to make measurements on laboratory test panels. As a simulation of outdoor weathering, the test panels were subjected to artificial weathering under ultraviolet light and water condensation cycles and natural outdoor weathering. Measurements were made using both standard fluid cells and co-planar hydrogel cells on both primer-only and primed \& painted substrates initially and after natural or artificial weathering. The EIS response of primer-only was studied in order to observe its degradation separately from that of paint \& primer. Before weathering, the primeronly coating was intact (having good barrier properties by EIS) and the EIS spectra obtained using either standard or co-planar hydrogel cells are comparable (Fig. 2.4A). SEM images of an initial primer-only sample (before weathering) is shown below in Figure 2.4A. The surface of the coating is smooth and free of obvious defects such as pores or cracks. A measurable sheet resistance was not obtainable by EIS at this initial time-point, suggesting that the sheet resistance was much greater than overall 
impedance of the bulk coating. After three months of natural outdoor weathering (Fig. 2.4B) the co-planar hydrogel cell spectrum deviated from the standard cell spectrum in the frequency range between 100 to $0.01 \mathrm{~Hz}$. The capacitive element at high frequencies (between 100 and $10^{6} \mathrm{~Hz}$ ) had the same value as the high frequency capacitive element shown in the standard cell measurement, which was fit with a CPE between $10^{4}$ and $10^{6}$ Hz. Based on the model fit values for the CPEs of $62.6 \pm 0.493 \mathrm{pS} \cdot \mathrm{s}^{\mathrm{a}} \cdot \mathrm{cm}^{-2} ; \alpha=0.937 \pm$ 0.000582 and of $43.1 \pm 0.771 \mathrm{pS} \cdot \mathrm{s}^{\mathrm{a}} \cdot \mathrm{cm}^{-2} ; \alpha=0.967 \pm 0.00132$ for the co-planar hydrogel and standard cell measurements respectively, the high frequency CPE element was assigned to the capacitance of the coating in both the Std and Cop cells. Importantly, at lower frequencies, a resistive element (the sheet resistance) was observed only in the co-planar hydrogel cell spectrum. When the hydrogels were lifted after taking a measurement, a darkening of the primer due to wetting was observed under and around the footprint of the hydrogels, which suggested diffusion of the electrolyte into the coating. It was the degradation of the surface of the coating during weathering that permitted such wetting to occur, forming a continuous, lateral pathway for current to travel. The resistive pathway was therefore assigned to the sheet resistance of the electrolyte saturated surface layer of the coating as seen in Figure 2.4B. The sheet resistance of naturally weathered primer decreased between three and four months from $47.7 \pm 2.42$ to $0.966 \pm 0.0238 \mathrm{M} \Omega / \square$ and slightly increased at six months to $1.83 \pm 0.00348 \mathrm{M} \Omega / \square$. The changes in sheet resistance between three and four months suggested either an increase in porosity of the surface of the coating or increase in thickness (depth) of the electrolyte saturated surface area, both of which would produce a lower resistance to current. For the four and six month time points, the sheet 
resistance values were similar to each other, and their slight difference can be explained by positioning of the hydrogels, where different areas of the coating would have slightly different porosity. The SEM image below the EIS spectra in Figure 2.4B supported the conclusion of the presence of a porous surface layer at six months, and its thickness decreased from an initial value of $204 \pm 6.9$ to $191 \pm 5.5 \mu \mathrm{m}$. The changes in overall coating thickness suggested that the porous layer was produced by a loss of material (rather than accumulation of debris on the surface). It is interesting to note that the overall impedance of the primer as measured with the standard cell increased between three and four months of weathering. Increases in impedance can be caused by a growth of an insulating corroded layer on the substrate such that the measured impedance is the sum of multiple capacitive layers (i.e. the coating and the underlying growing corrosion layer). That was most likely the case for the EIS measurements of the primer alone, as primer only is not intended to be protective over time without a topcoat of paint. In this case, the sheet resistance is indicative of the protective state of the primer as it indicated the beginning of primer degradation at the surface level.

A measurable sheet resistance was also observed in the primer \& paint substrate after natural weathering. The paint did not initially have a measureable sheet resistance in the co-planar hydrogel measurements (Fig 2.4D) similarly to the primer. Below the EIS spectra, in Figure 2.4D, the SEM image of an initial painted plate shows a somewhat bumpy surface that is free of obvious defects. A measurable sheet resistance was then present at four and six months of natural weathering (Fig. 2.4E). When sheet resistance was first observed in the paint after four months of natural weathering, it is a much higher value $(1,140 \pm 169 \mathrm{M} \Omega / \square)$ than the initial sheet resistance in the primer- 
only substrate $(47.7 \pm 2.42 \mathrm{M} \Omega / \square)$. This was expected, as the surface of the painted panel should be more resistant to degradation over time than the primer-only surface and therefore diffusion of electrolyte across the surface would not occur until later time points and when it occurs, the resistance value would be greater. After six months of natural weathering, the sheet resistance stayed the same as the four month measurement, at $1,120 \pm 221 \mathrm{M} \Omega / \square$ and its surface can be seen by SEM below the EIS spectra in in Figure 2.4E. The surface is free of visible defects but the presence of a measurable sheet resistance suggested that degradation of the surface was, in fact, beginning to occur. The coating thickness did not significantly change from the initial time-point of weathering ( $178 \pm 3.7$ to $174 \pm 4.0 \mu \mathrm{m})$, however the bumps present within the SEM image in Figure 2.4D were no longer present, supporting that the structure had changed somewhat. At this point in weathering, the standard cell EIS data suggested that the bulk of the coating remained protective and the co-planar hydrogel EIS data supported the bulk observations as well provided information that surface was just starting to degrade.

Primer-only and primer \& paint samples were also weathered artificially in a QUV-B chamber. A measurable sheet resistance was observed in primer-only after it was artificially aged for 250 hours (Fig. 2.4C). Artificially weathered primer-only decreased in sheet resistance value from $608 \pm 62.0$ to $235 \pm 4.98 \mathrm{k} \Omega / \square$ between 250 and 1000 hours. At 1000 hours, the artificially aged primer-only decreased in thickness from $221 \pm 7.6$ to $205 \pm 9.1 \mu \mathrm{m}$ respectively. The SEM image of primer-only at 1000 hours below the EIS spectra in Figure 2.4C shows a surface with large pores. A measureable sheet resistance was not present in the paint plus primer sample until 1000 
hours of artificial weathering (Fig 2.4F) and the value was similar to that of the primeronly at 250 hours $(256 \pm 31.6$ and $608 \pm 62.0 \mathrm{k} \Omega / \square$, respectively). The SEM image below the EIS spectra in Figure 2.4F shows the damaged surface of the paint after 1000 hours of artificial weathering; and the coating thickness decreased from $196 \pm 4.0$ initially to $176 \pm 7.2 \mu \mathrm{m}$ after $1000 \mathrm{~h}$. At that time point, both the primer-only and paint \& primer could still be considered to be protective according to standard cell EIS data, but the co-planar hydrogel cell revealed significant degradation of the surface layers of the coatings. With the separation along the frequency axis between coating capacitance (present at high frequencies) and sheet resistance (present at low frequencies), information about both the bulk of the coating and surface of the coating is available through co-planar hydrogel measurements. Both the presence and magnitude of sheet resistance provide information about the state of the surface of the coating. Because measurements made using the co-planar hydrogel cell provide more superficial information compared to the bulk type of information from the standard cell, this measurement type therefore offers an earlier warning sign of degradation as coatings age due to top-down solar illumination and oxidative damage. 


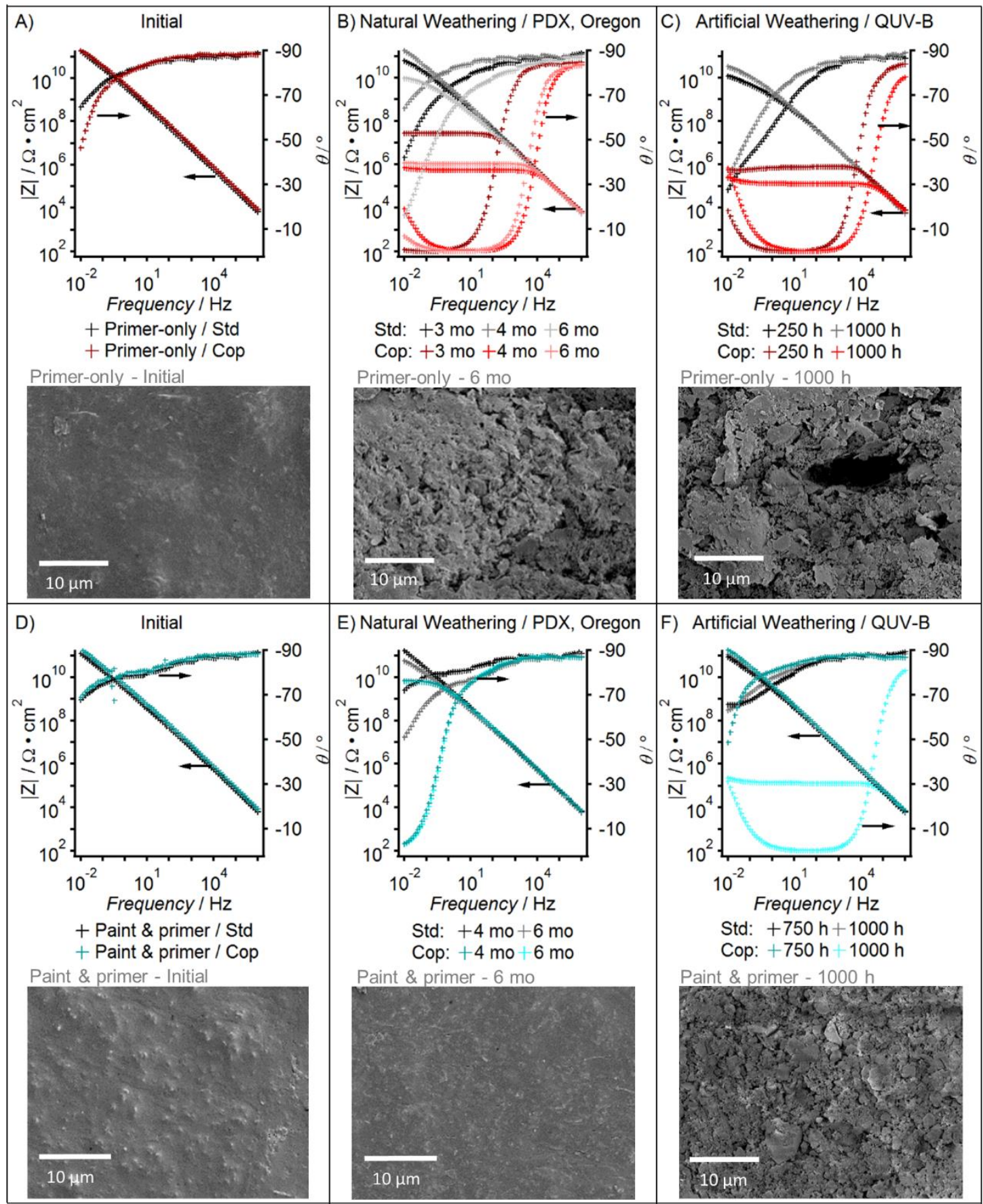

Figure 2.4 Standard cell (Std) and co-planar (Cop) EIS measurements and a corresponding SEM image at a specified time point of primer-only A) initially, (B) after 3, 4, and 6 months of natural weathering in Portland, Oregon, and C) after 250 and 1000 hours of artificial weathering in a QUV-B chamber and paint \& primer D) initially, E) after 4 and 6 months of natural weathering in Portland, Oregon and F) after 750 and 1000 hours of artificial weathering in a QUV-B chamber. EIS spectra are normalized to the area underneath the co-planar hydrogels. Spectra including the sheet resistance element are overlaid for the purpose of illustrating the deviation from standard liquid cell and trend in resistance over time and the resistive portion of the plots are not representative of normalized sheet resistance listed in the text. 


\subsubsection{In situ measurements}

\subsubsection{Co-planar hydrogel vs. standard cell at The Olympic Sculpture Park}

Co-planar hydrogel and standard cell EIS measurements were compared at the Seattle Art Museum's Olympic Sculpture Park in Seattle, Washington. Tony Smith's Stinger is a hollow steel sculpture that is coated with the same primer and paint as our in-lab measurements described above. The sculpture includes a flat, removable access panel that was coated on the inward-facing side with primer-only. The panels also had non-coated bolt holes that allowed for standard cell measurements to be performed by attaching the working electrode to the threads within one of those holes. Thus, this panel allowed the comparison of EIS data between both standard and co-planar hydrogel cells to be made, demonstrating the validity of the measurement method and producing data from the field. The data from these field measurements are displayed in Figure 2.5A; and the two spectra are comparable in shape. The slight difference in impedance can be explained by the different area measured by the two cell types. The co-planar hydrogel cell measures a smaller area and is therefore more sensitive to defects in the coating, explaining the slightly lower impedance at mid-range frequencies. These data offer further confirmation that the co-planar hydrogel cell gives similar information when compared to the standard cell when sheet resistance is able to be measured. Because sheet resistance was not observed by EIS, it can be said that the primer is still in a very protective state as this suggests the surface of the coating has not yet started to degrade. This was expected as the measured primer-only area is facing inward on the sculpture and is therefore protected from outside elements such as water, pollutants, and UV rays. 


\subsubsection{Sheet resistance at The Olympic Sculpture Park}

On all other parts of the Stinger, standard cell EIS measurements could not be made, due to an absence of non-coated metal substrate to attach the working electrode. Instead, co-planar hydrogel EIS measurements of several painted portions of Stinger were obtained. Multiple measurements were taken from two separate regions on the sculpture (Fig. 2.5B) and the spectra are displayed in Figure 2.5C. An open lead measurement was performed which provides the maximum measureable impedance of the potentiostat within the specified frequency range. The open lead spectrum overlaid
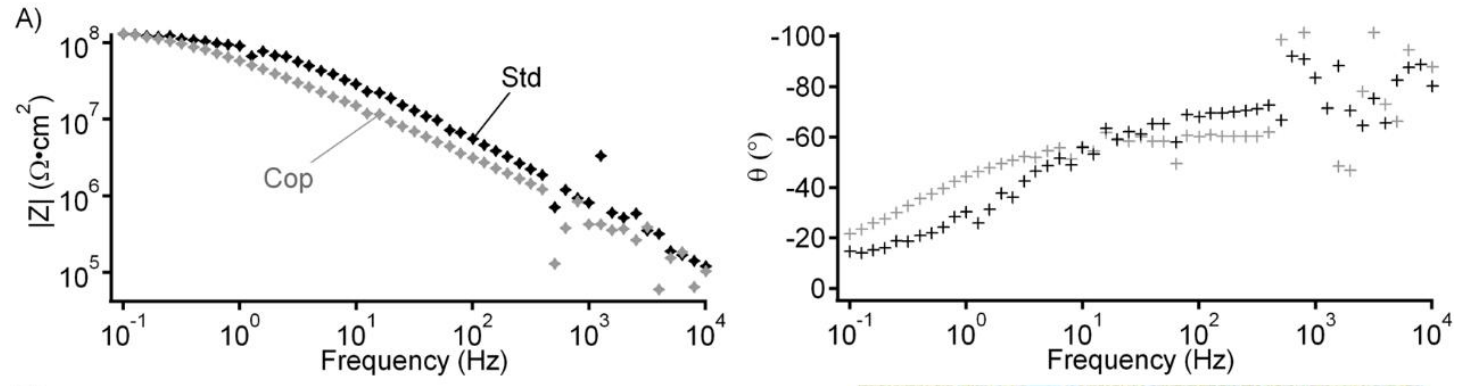

B)
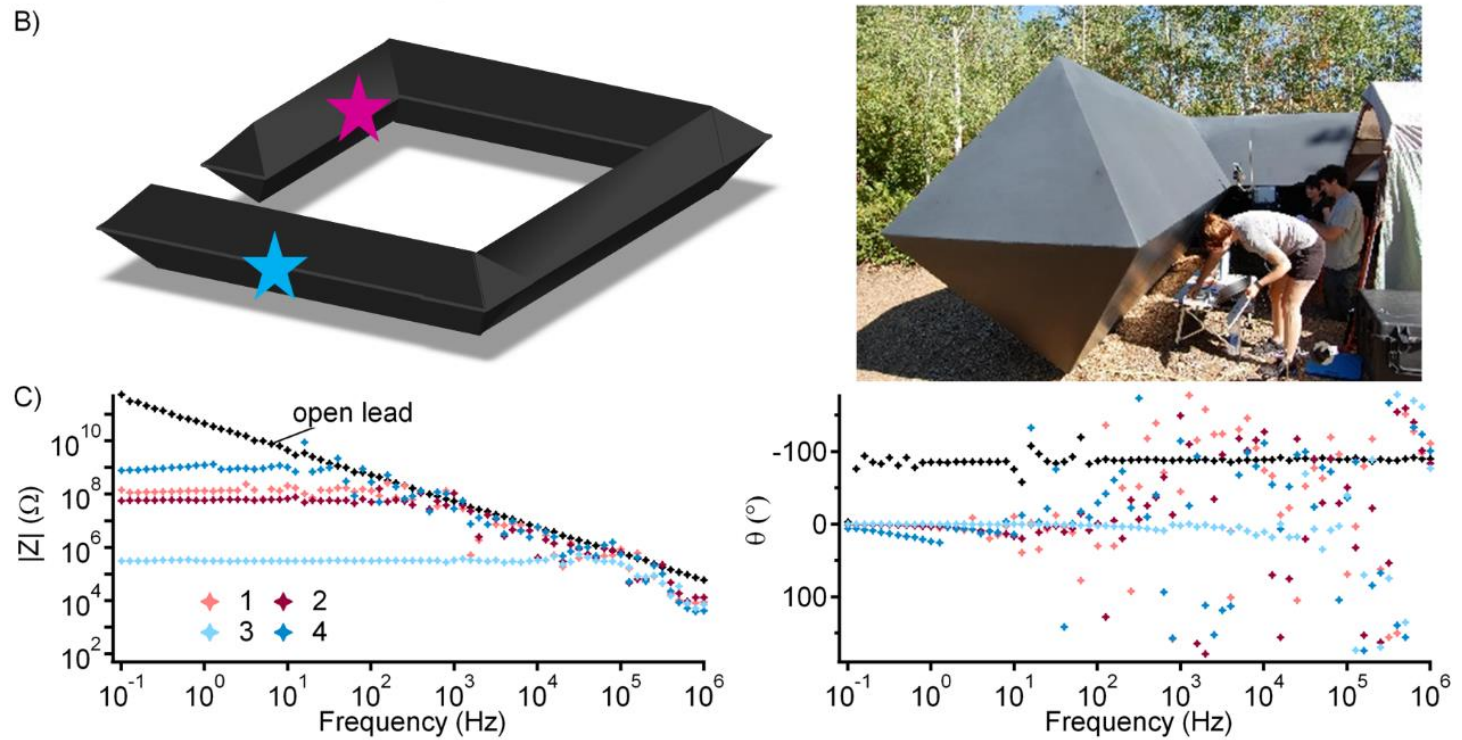

Figure 2.5 A) Standard cell and co-planar measurements on an access panel from Tony Smith's Stinger B) An illustration and photo of Stinger by Tony Smith with the regions of co-planar measurements are highlighted by the corresponding colored stars. C) EIS spectra from co-planar measurements on the isolated regions on Stinger. 
with spectra from Stinger showed that the impedance due to the capacitance of the coating is greater than the measurable limit of the instrument. At lower frequencies, a measurable sheet resistance is present. Meaurements from areas 1 and 2 were from the same general region on Stinger and, predictably, show a similar impedance. Measurements from areas 3 and 4 were taken from another region on the sculpture and, surprisingly, the measured impedances differed over an order of magnitude.

The sheet resistances of the four areas on Stinger were calculated and are shown in Figure 2.6 along with the calculated sheet resistances of the primer-only and paint \& primer weathered in the lab. Areas 1,2 , and 4 show similar resistances to the paint \& primer samples at either four or six months of natural weathering in Portland, OR and suggests that the surfaces may be similar in appearance to that shown in Figure 2.4E. The high value of the sheet resistance is evidence that, while surface degradation is present, it in the beginning stages and the coating is still very protective in nature. Area 3 has a measurable sheet resistance comparable to primer-only that had been naturally weathered outdoors for six months. This suggests that the surface of the coating is similar to that of Figure $2.4 \mathrm{~B}$ and is in later stages of degradation. From these data, it is evident that area 3 of Stinger could be locally treated while areas 1, 2, and 4 were sufficiently intact as the time they were measured; and re-coating of the entire sculpture was not needed. Periodic monitoring of the sculpture by our method would be able determine when it is recommended to re-coat the entire sculpture. 


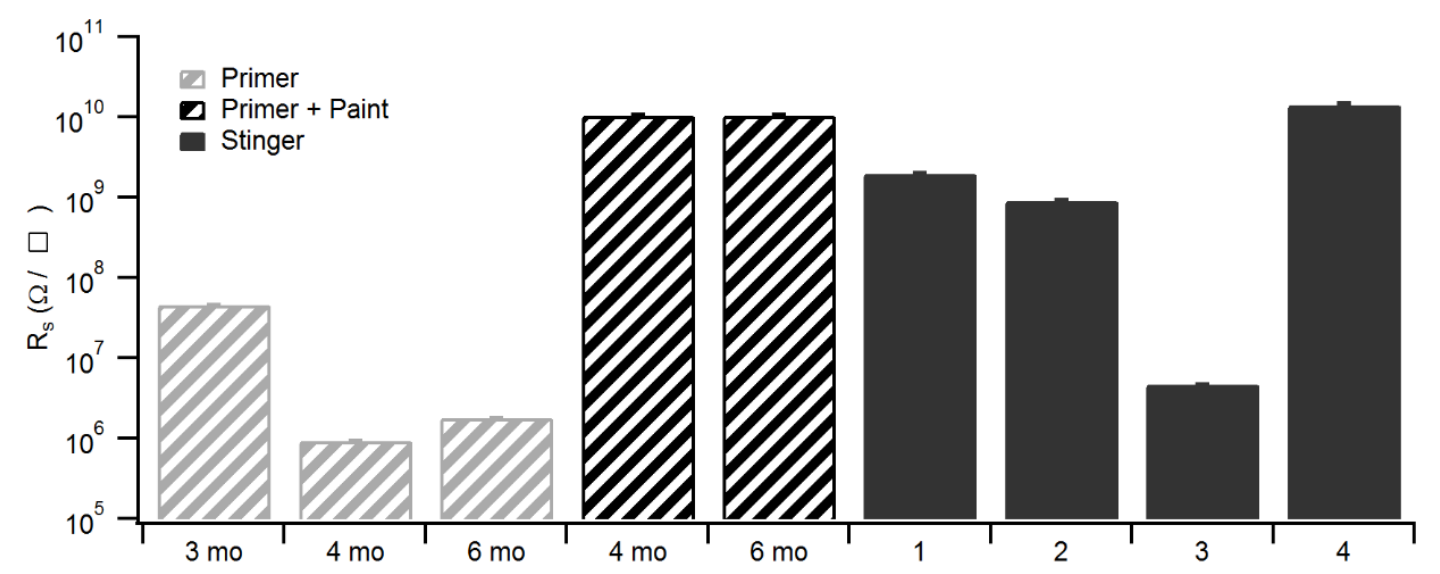

Figure 2.6 Sheet resistances of measurements on weathered primer-only, primer \& paint, and Stinger.

\subsection{Conclusions}

Several experiments were performed to explore sheet resistance as a measure of coating degradation as well as to elucidate the differences between the co-planar hydrogel and standard cell types for EIS data collection. Sheet resistance was found to be a measureable variable indicative of early warning signs of coating degradation before such signs of bulk coating failure are present and measurable. Having a method capable of distinguishing superficial from bulk degradation is critical in preventing corrosion processes from starting. Both co-planar hydrogel and standard cell types are able to measure properties of the bulk of a protective coating through a direct pathway that travels through the cross-section of a coating to the metal substrate, while only the co-planar hydrogel cell is also able to measure a lateral pathway through the surface of a coating to obtain sheet resistance data - thus it offers more information than the conventional cell type does. If the system impedance is in the measurable range and only information about the bulk of the coating is needed, it is possible to alter the coplanar hydrogel cell by applying pressure around the hydrogels to force the direct 
pathway - thus our novel co-planar hydrogel cell can be tailored to obtain the coating properties that are wanted. Since hydrogels are conformable and are not liquids, the substrates that can be measured using them may be non-planar, the surfaces need not be horizontal and the size of the cells are easily variable - thus enabling information to be obtained on a considerable variety of substrates. Lastly, because this measurement method cell set-up is non-destructive, it offers a viable measurement for in-field analysis of protective coatings on both artistic and architectural metalwork. Conventionally, the protective properties of coatings are assumed by proxy from mockup test panels and not actually measured in situ where exposure conditions and application of the coatings may develop vastly different properties during aging compared to the aging profiles of test panels. 


\section{References}

1. del Valle, M., Electronic Tongues Employing Electrochemical Sensors. Electroanalysis 2010, 22 (14), 1539-1555.

2. Mansfeld, F., Use of electrochemical impedance spectroscopy for the study of corrosion protection by polymer coatings. Journal of Applied Electrochemistry 1995, 25 (3), 187-202.

3. Macdonald, D. D., Reflections on the history of electrochemical impedance spectroscopy. Electrochim. Acta 2006, 51 (8-9), 1376-1388.

4. Chang, B.-Y.; Park, S.-M., Electrochemical Impedance Spectroscopy. Annual Review of Analytical Chemistry 2010, 3 (1), 207-229.

5. Grundmeier, G.; Schmidt, W.; Stratmann, M., Corrosion protection by organic coatings: electrochemical mechanism and novel methods of investigation. Electrochim. Acta 2000, 45 (15-16), 2515-2533.

6. Lavaert, V.; De Cock, M.; Moors, M.; Wettinck, E., Influence of pores on the quality of a silicon polyester coated galvanised steel system. Prog. Org. Coat. 2000, 38 (3-4), 213-221.

7. Lavaert, V.; Moors, M.; Wettinck, E., An EIS study of the influence of imperfections on the corrosion behaviour of an organic coated steel system. Journal of Applied Electrochemistry 2002, 32 (8), 853-857.

8. Curkovic, H. O.; Kosec, T.; Marusic, K.; Legat, A., An electrochemical impedance study of the corrosion protection of artificially formed patinas on recent bronze. Electrochim. Acta 2012, 83, 28-39.

9. Swartz, N. A.; Clare, T. L., Understanding the differences in film formation mechanisms of two comparable solvent based and water-borne coatings on bronze substrates by electrochemical impedance spectroscopy. Electrochim. Acta 2011, 62, 199-206.

10. Le Thu, Q.; Takenouti, H.; Touzain, S., EIS characterization of thick flawed organic coatings aged under cathodic protection in seawater. Electrochim. Acta 2006, 51 (12), 2491-2502.

11. van der Weijde, D. H.; van Westing, E. P. M.; de Wit, J. H. W., EIS measurements on artificial blisters in organic coatings. Electrochim. Acta 1996, 41 (7-8), 1103-1107.

12. Cano, E.; Lafuente, D.; Bastidas, D., Use of EIS for the evaluation of the protective properties of coatings for metallic cultural heritage: a review. Journal of Solid State Electrochemistry 2009, 14 (3), 381-391.

13. Amand, S.; Musiani, M.; Orazem, M. E.; Pébère, N.; Tribollet, B.; Vivier, V., Constant-phase-element behavior caused by inhomogeneous water uptake in anticorrosion coatings. Electrochim. Acta 2013, 87 (0), 693-700.

14. Bonora, P. L.; Deflorian, F.; Fedrizzi, L., Electrochemical Impedance Spectroscopy as a tool for investigating underpaint corrosion. Electrochim. Acta 1996, 41 (7-8), 1073-1082.

15. Rammelt, U.; Reinhard, G., Characterization of active pigments in damage of organic coatings on steel by means of electrochemical impedance spectroscopy. Prog. Org. Coat. 1994, 24 (1-4), 309-322. 
16. Schwake, A.; Geuking, H.; Cammann, K., Application of a new graphical fitting approach for data analysis in electrochemical impedance spectroscopy. Electroanalysis 1998, 10 (15), 1026-1029.

17. Hirayama, R.; Haruyama, S., Electrochemical Impedance for Degraded Coated Steel Having Pores. Corrosion 1991, 47 (12), 952-958.

18. Tsai, C. H.; Mansfeld, F., Determination of Coating Deterioration with EIS: Part II. Development of a Method for Field Testing of Protective Coatings. Corrosion 1993, 49 (9), 726-737.

19. Mahdavian, M.; Attar, M. M., Another approach in analysis of paint coatings with EIS measurement: Phase angle at high frequencies. Corros. Sci. 2006, 48 (12), 4152-4157.

20. Zhao, X.; Wang, J.; Wang, Y.; Kong, T.; Zhong, L.; Zhang, W., Analysis of deterioration process of organic protective coating using EIS assisted by SOM network. Electrochem. Commun. 2007, 9 (6), 1394-1399.

21. Xu, A. T.; Zhang, F.; Luo, B.; Jin, F.; Zhang, T. R., Investigation the Protective Performance of Organic Coatings with Different Breakage Degree Using EIS United to SOM Neural Network. Int. J. Electrochem. 2013, 8 (2), 1895-1902.

22. Xu, A. T.; Zhang, F.; Luo, B.; Jin, F.; Zhang, T. R., Investigation the Deterioration Process of Organic Coating Using Changing Rate of Phase Angle at High Frequency United to Neural Network. Int. J. Electrochem. 2013, 8 (1), 773 779.

23. Akbarinezhad, E.; Bahremandi, M.; Faridi, H. R.; Rezaei, F., Another approach for ranking and evaluating organic paint coatings via electrochemical impedance spectroscopy. Corros. Sci. 2009, 51 (2), 356-363.

24. Kouhi, M.; Mohebbi, A.; Mirzaei, M., Evaluation of the corrosion inhibition effect of micro/nanocapsulated polymeric coatings: a comparative study by use of EIS and Tafel experiments and the area under the Bode plot. Research on Chemical Intermediates 2013, 39 (5), 2049-2062.

25. Lazzari, M.; Chiantore, O., Thermal-ageing of paraloid acrylic protective polymers. Polymer 2000, 41 (17), 6447-6455.

26. Hinderliter, B. R.; Croll, S. G., Simulation of transient electrochemical impedance spectroscopy due to water uptake or oxide growth. Electrochim. Acta 2009, 54 (23), 5344-5352.

27. Kumar, G.; Buchheit, R. G., Use of artificial neural network models to predict coated component life from short-term electrochemical impedance spectroscopy measurements. Corrosion 2008, 64 (3), 241-254.

28. Miszczyk, A.; Darowicki, K., Multispectral impedance quality testing of coilcoating system using principal component analysis. Prog. Org. Coat. 2010, 69 (4), 330-334.

29. G.H. Koch; Brongers, M. P. H.; Thompson, N. G.; Virmani, Y. P.; Payer, J. H. Corrosion Costs and Preventive Strategies in the United States; FHWA-RD-01156; U.S. Department of Transportation, Federal Highway Administration: 2002.

30. Leidheiser, H., Corrosion of Painted Metals_A Review. Corrosion 1982, 38 (7), 374-383. 
31. Funke, W., Problems and progress in organic coatings science and technology. Prog. Org. Coat. 1997, 31 (1-2), 5-9.

32. Clare, T. L.; Swartz, N. A., Chapter 19 - Characterization of High Performance Protective Coatings for Use on Culturally Significant Works A2 - Tiwari, Atul. In Intelligent Coatings for Corrosion Control, Rawlins, J.; Hihara, L. H., Eds. Butterworth-Heinemann: Boston, 2015; pp 641-671.

33. Barsoukov, E.; Macdonald, J. R., Impedance Spectroscopy: Theory, Experiment, and Applications. Wiley, Interscience: Hoboken, NJ, 2005.

34. Cano, E.; Bastidas, D. M.; Argyropoulos, V.; Fajardo, S.; Siatou, A.; Bastidas, J. M.; Degrigny, C., Electrochemical characterization of organic coatings for protection of historic steel artefacts. Journal of Solid State Electrochemistry 2010, $14(3), 453-463$.

35. Ellingson, L. A.; Shedlosky, T. J.; Bierwagen, G. P.; Rie, E. R. d. 1.; Brostoff, L. B., The Use of Electrochemical Impedance Spectroscopy in the Evaluation of Coatings for Outdoor Bronze. Studies in Conservation 2004, 49 (1), 53-62.

36. Hosbein, K. N.; Swartz, N. A.; Clare, T. L., Electrochemical Identification and Categorization of the Protective Quality of Intact and Damaged Coatings. Electroanalysis 2014, 26 (9), 1935-1944.

37. Perrin, F. X.; Merlatti, C.; Aragon, E.; Margaillan, A., Degradation study of polymer coating: Improvement in coating weatherability testing and coating failure prediction. Prog. Org. Coat. 2009, 64 (4), 466-473.

38. Zuo, Y.; Zhang, L.; Zhao, X.; Tang, Y.; Zhang, X., Failure process of acrylic polyurethane coating under alternate wetting and drying condition. Journal of Applied Polymer Science 2015, 132 (20).

39. Skale, S.; Dolecek, V.; Slemnik, M., Electrochemical impedance studies of corrosion protected surfaces covered by epoxy polyamide coating systems. Prog. Org. Coat. 2008, 62 (4), 387-392.

40. Ramirez Barat, B.; Cano, E., The use of agar gelled electrolyte for in situ electrochemical measurements on metallic cultural heritage. Electrochim. Acta 2015, 182, 751-762.

41. Angelini, E.; Grassini, S.; Corbellini, S.; Ingo, G. M.; de Caro, T.; Plescia, P.; Riccucci, C.; Bianco, A.; Agostini, S., Potentialities of XRF and EIS portable instruments for the characterisation of ancient artefacts. Applied Physics A: Materials Science \& Processing 2006, 83 (4), 643-649.

42. Angelini, E.; Grassini, S.; Parvis, M.; Zucchi, F., An in situ investigation of the corrosion behaviour of a weathering steel work of art. Surface and Interface Analysis 2011, 44 (8), 942-946.

43. Corbellini, S.; Parvis, M.; Grassini, S., Noninvasive Solution for Electrochemical Impedance Spectroscopy on Metallic Works of Art. IEEE Trans. Instrum. Meas. 2012, 61 (5), 1193-1200.

44. Corbellini, S.; Parvis, M.; Grassini, S.; Ieee, Development and characterization of flexible electrodes for protective painting monitoring. In 2011 Ieee International Instrumentation and Measurement Technology Conference, 2011; pp 1530-1535. 
45. England, A. H.; Clare, T. L., Synthesis and Characterization of Flexible Hydrogel Electrodes for Electrochemical Impedance Measurements of Protective Coatings on Metal Sculptures. Electroanalysis 2014, 26 (5), 1059-1067.

46. Clare, T. L.; England, A. H.; Swartz, N. A.; K. N. Hosbein, ICOM Metals 2013 Proc. . 2013; pp 89-94. 
Appendix: Previously published methods for quick analysis and KramersKronig transformations

\section{A.1 Comparison with previously published methods for quick analysis of impedance data}

Previous studies have investigated the issue of identifying methods to more quickly analyze impedance data that do not require circuit modelling. In this supplementary information, we present a comparison between the categories determined by the impedance of discrete elements representative of failing coatings, (derived from circuit modeling) and six previously published methods. Using the same twenty test panels, correlation plots of the published method versus the circuit modelling result were produced and are shown in Figure A.1. A summary of the calculated values from the published methods of quicker analysis are compiled in Tables A.1 and A.2. 


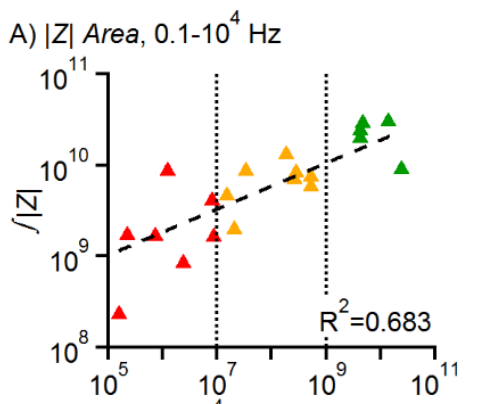

D) $R_{h}, \mathrm{f}=100-10^{4} \mathrm{~Hz}$

B) \% Decrease $|\mathrm{Z}|$ Area, $0.1-10^{4} \mathrm{~Hz}$
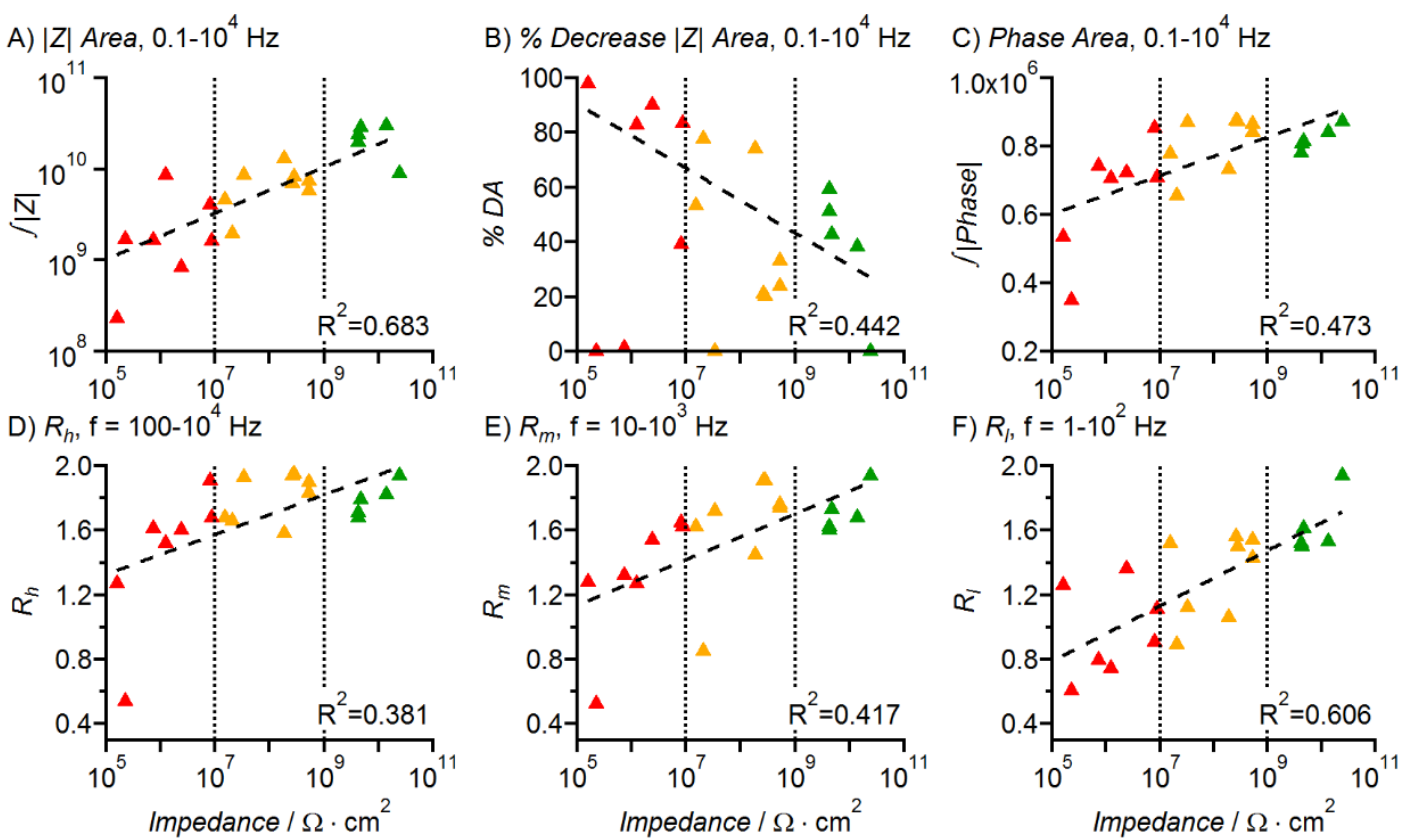

E) $R_{m}, \mathrm{f}=10-10^{3} \mathrm{~Hz}$

F) $R, f=1-10^{2} \mathrm{~Hz}$
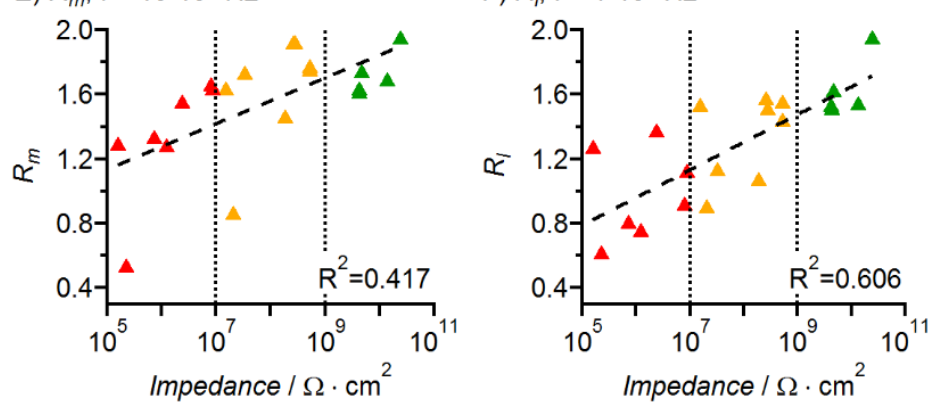

Figure A.1 EEC classification vs A) integrated impedance modulus $|Z|$ area: $y=3 E+07 x^{0.282} R^{2}=0.683$; B) relative change in bode area after weathering \%DA: $y=-5.137 \ln (x)+149.84, R^{2}=0.442$; $)$ integrated Phase Area: $y=24461 \ln (x)+319836, R^{2}=0.473$; D) ratio of high frequency impedance at $100 \mathrm{~Hz}$ and $\left.10 \mathrm{kHz} \mathrm{R}_{\mathrm{h}}: \mathrm{y}=0.0536 \ln (\mathrm{x})+0.7107, \mathrm{R}^{2}=0.381 ; \mathrm{E}\right)$ ratio of midrange frequency impedance at $10 \mathrm{~Hz}$ and $1 \mathrm{kHz} \mathrm{R}$ : $: y=0.0615 \ln (x)+0.4264, \mathrm{R}^{2}=0.417$; and $\mathrm{F}$ ) ratio of low frequency impedance at $1 \mathrm{~Hz}$ and $100 \mathrm{~Hz} \mathrm{R}$ : $\mathrm{y}=0.0745 \ln (\mathrm{x})-0.0687, \mathrm{R}^{2}=0.606$. The vertical dotted lines indicate the category boundaries determined by circuit models and the color $($ green $=\mathrm{E}$, yellow=A, red $=\mathrm{P}$ ) indicates the protective quality of each coated sample. Corresponding horizontal lines for the category boundaries could not be determined due to the overlap of coatings having different protective qualities.

\section{A.1.1 Calculations}

Areas of the $\mathrm{Z}$ modulus and phase angle plots as described in a previously published study ${ }^{23}$ using Simpson's trapezoidal area approximations and the percentage of the $\mathrm{Z}$ modulus plot were calculated as follows:

$$
\begin{gathered}
\int_{x_{0}}^{x_{n}} f(x) d x=\frac{\Delta x}{2}\left[f\left(x_{0}\right)+2 f\left(x_{1}\right)+\cdots+2 f\left(x_{n-1}\right)+f\left(x_{n)}\right]\right. \\
\% D A=\frac{100 x\left(\varphi_{0}-\varphi_{t}\right)}{\varphi_{0}}
\end{gathered}
$$


Where DA is the decrease in area of the $\mathrm{Z}$ modulus plot in percent, $\varphi_{0}$ is the initial area before weathering/damage and $\varphi_{\mathrm{t}}$ is the integrated area after weathering/damage at time $(t)$. The ratios of two impedance modulus values have been described to more quickly analyze impedance data in previously published work by others ${ }^{18}$. Using their methods here, we calculated the ratios at three different frequency ranges: at high $\left(R_{h}\right)$, mid $\left(R_{m}\right)$ and low $\left(R_{l}\right)$ as follows:

$$
\begin{aligned}
& R_{h}=\log \frac{|Z|_{100 \mathrm{~Hz}}}{|Z|_{10 \mathrm{kHz}}} \\
& R_{m}=\log \frac{|Z|_{10 \mathrm{~Hz}}}{|Z|_{1 \mathrm{kHz}}} \\
& R_{l}=\log \frac{|Z|_{1 \mathrm{~Hz}}}{|Z|_{100 \mathrm{~Hz}}}
\end{aligned}
$$

where $R$ denotes a ratio, the subscripts $h, m$, and $l$ indicate which portion of the bode plot the values were obtained, and $|Z|_{f}$ is the impedance measured at the respective frequency. 
Table A.1 Values of parameters determined from EIS spectra used in the categorization of the protective quality of coatings for EEC, impedance magnitude area, and the decrease in Bode impedance magnitude area percentage methods. The frequency range used in each method is listed below the column heading. The protective quality determined by each method is given by: excellent $(\mathrm{E})$, adequate $(\mathrm{A})$, and poor $(\mathrm{P})$.

\begin{tabular}{|c|c|c|c|c|c|}
\hline $\begin{array}{l}\text { Coating } \\
\text { Type }\end{array}$ & Weathering & $\begin{array}{l}\text { Model/ } \\
\text { Element }\end{array}$ & $\begin{array}{c}\text { Element Impedance } \\
\left(\Omega \cdot \mathrm{cm}^{2}\right)\end{array}$ & |Z| Area & $\begin{array}{c}\text { Decrease in } \\
\text { Bode Area \% }\end{array}$ \\
\hline & & & $0.1-1 \times 10^{6} \mathrm{~Hz}$ & $0.1-10^{4} \mathrm{~Hz}$ & $0.1-10^{4} \mathrm{~Hz}$ \\
\hline 1 & None & $1 / \mathrm{R}_{1}$ & $2.40 \times 10^{10}(\mathrm{E})$ & $8.86 \times 10^{9}$ & $\mathrm{n} / \mathrm{a}+$ \\
\hline 1 & None & $2 b / R_{1}$ & $3.29 \times 10^{7}(\mathrm{~A})$ & $2.99 \times 10^{10}$ & 38.2 \\
\hline 1 & Abrasion & $2 \mathrm{~b} / \mathrm{R}_{1}$ & $2.58 \times 10^{8}(\mathrm{~A})$ & $2.84 \times 10^{10}$ & 42.8 \\
\hline 1 & MPD* & $3 / \mathrm{CPE}_{3}$ & $1.57 \times 10^{5}(\mathrm{P})$ & $2.36 \times 10^{10}$ & 51.2 \\
\hline 1 & FL 9 mo & $3 \mathrm{~b} / \mathrm{CPE}_{3}$ & $7.95 \times 10^{6}(\mathrm{P})$ & $1.97 \times 10^{10}$ & 59.3 \\
\hline 1 & QUV-B $500 \mathrm{~h}$ & $2 \mathrm{~b} / \mathrm{R}_{1}$ & $2.78 \times 10^{8}(\mathrm{~A})$ & $5.82 \times 10^{9}$ & 33.1 \\
\hline 1 & QUV-B $500 \mathrm{~h}$ & $3 / \mathrm{CPE}_{3}$ & $2.36 \times 10^{6}(\mathrm{P})$ & $7.49 \times 10^{9}$ & 23.9 \\
\hline 2 & PDX 15 mo & $2 / \mathrm{R}_{2}$ & $5.28 \times 10^{8}(\mathrm{~A})$ & $8.25 \times 10^{9}$ & 20.2 \\
\hline 2 & PDX 31 mo & $2 / \mathrm{R}_{2}$ & $2.02 \times 10^{7}(\mathrm{~A})$ & $7.00 \times 10^{9}$ & 21 \\
\hline 2 & QUV-B $500 \mathrm{~h}$ & $2 / R_{2}$ & $5.18 \times 10^{8}(\mathrm{~A})$ & $1.29 \times 10^{10}$ & 74 \\
\hline 2 & QUV-B $1000 \mathrm{~h}$ & $3 \mathrm{~b} / \mathrm{CPE}_{3}$ & $1.52 \times 10^{7}(\mathrm{~A})$ & $1.94 \times 10^{9}$ & 77.7 \\
\hline 2 & QUV-B $1500 \mathrm{~h}$ & $3 \mathrm{~b} / \mathrm{CPE}_{3}$ & $8.64 \times 10^{6}(\mathrm{P})$ & $4.61 \times 10^{9}$ & 53.2 \\
\hline 3 & None & $2 \mathrm{~b} / \mathrm{R}_{1}$ & $2.23 \times 10^{5}(\mathrm{P})$ & $8.59 \times 10^{9}$ & $\mathrm{n} / \mathrm{a}+$ \\
\hline 3 & QUV-B $1250 \mathrm{~h}$ & $3 / \mathrm{R}_{2}$ & $7.24 \times 10^{5}(\mathrm{P})$ & $1.63 \times 10^{9}$ & 83.4 \\
\hline 4 & $20 \mathrm{~h}$ soak & $1 / R_{1}$ & $4.64 \times 10^{9}(\mathrm{E})$ & $4.05 \times 10^{9}$ & 39.3 \\
\hline 4 & $68 \mathrm{~h}$ soak & $2 / \mathrm{R}_{2}$ & $1.86 \times 10^{8}(\mathrm{~A})$ & $8.41 \times 10^{8}$ & 90.2 \\
\hline 4 & 8 day soak & $3 / \mathrm{R}_{2}$ & $1.23 \times 10^{6}(\mathrm{P})$ & $8.60 \times 10^{9}$ & 82.7 \\
\hline 5 & $25 \mathrm{~h}$ soak & $2 / R_{2}$ & $1.36 \times 10^{10}(\mathrm{E})$ & $1.67 \times 10^{9}$ & $1.3 \%$ \\
\hline 5 & 7 day soak & $2 / \mathrm{R}_{2}$ & $4.28 \times 10^{9}(\mathrm{E})$ & $1.69 \times 10^{9}$ & $\mathrm{n} / \mathrm{a}$ \\
\hline 5 & 62 day soak & $2 / R_{2}$ & $4.23 \times 10^{9}(\mathrm{E})$ & $2.29 \times 10^{8}$ & 98 \\
\hline
\end{tabular}

*multiple point defects, soak $=$ immersion in electrolyte, $\$$ denotes samples that were incorrectly categorized by that method. 
Table A.2 Values of parameters determined from EIS spectra used in the categorization of the protective quality of coatings for Bode phase area, and ratio of change in Bode impedance magnitude for high, low, and mid range frequencies. The frequency range used in each method is listed below the column heading. The protective quality determined by each method is given by: excellent (E), adequate (A), and poor (P).

\begin{tabular}{|c|c|c|c|c|c|c|}
\hline \multirow{2}{*}{$\begin{array}{l}\text { Coating } \\
\text { Type }\end{array}$} & \multirow[t]{2}{*}{ Weathering } & \multirow{2}{*}{$\begin{array}{l}\text { Model/ } \\
\text { Element }\end{array}$} & |Phase Area| & $\mathrm{Rh}$ & $\mathrm{Rl}$ & $\mathrm{Rm}$ \\
\hline & & & & & & $10 \& 10^{3} \mathrm{~Hz}$ \\
\hline 1 & None & $1 / R_{1}$ & $8.72 \times 10^{5}$ & 1.940 & 1.940 & 1.940 \\
\hline 1 & None & $2 \mathrm{~b} / \mathrm{R}_{1}$ & $8.41 \times 10^{5}$ & 1.820 & 1.530 & 1.680 \\
\hline 1 & Abrasion & $2 \mathrm{~b} / \mathrm{R}_{1}$ & $8.15 \times 10^{5}$ & 1.790 & 1.610 & 1.730 \\
\hline 1 & MPD* & $3 / \mathrm{CPE}_{3}$ & $8.07 \times 10^{5}$ & 1.710 & 1.500 & 1.600 \\
\hline 1 & FL 9 mo & $3 \mathrm{~b} / \mathrm{CPE}_{3}$ & $7.81 \times 10^{5}$ & 1.680 & 1.520 & 1.620 \\
\hline 1 & QUV-B $500 \mathrm{~h}$ & $2 \mathrm{~b} / \mathrm{R}_{1}$ & $8.40 \times 10^{5}$ & 1.830 & 1.540 & 1.740 \\
\hline 1 & QUV-B $500 \mathrm{~h}$ & $3 / \mathrm{CPE}_{3}$ & $8.66 \times 10^{5}$ & 1.900 & 1.430 & 1.760 \\
\hline 2 & PDX 15 mo & $2 / \mathrm{R}_{2}$ & $8.73 \times 10^{5}$ & 1.950 & 1.500 & 1.910 \\
\hline 2 & PDX 31 mo & $2 / \mathrm{R}_{2}$ & $8.74 \times 10^{5}$ & 1.940 & 1.560 & 1.910 \\
\hline 2 & QUV-B $500 \mathrm{~h}$ & $2 / R_{2}$ & $7.32 \times 10^{5}$ & 1.580 & 1.060 & 1.450 \\
\hline 2 & QUV-B $1000 \mathrm{~h}$ & $3 \mathrm{~b} / \mathrm{CPE}_{3}$ & $6.56 \times 10^{5}$ & 1.660 & 0.894 & 0.851 \\
\hline 2 & QUV-B $1500 \mathrm{~h}$ & $3 \mathrm{~b} / \mathrm{CPE}_{3}$ & $7.79 \times 10^{5}$ & 1.680 & 1.520 & 1.620 \\
\hline 3 & None & $2 \mathrm{~b} / \mathrm{R}_{1}$ & $8.70 \times 10^{5}$ & 1.930 & 1.120 & 1.720 \\
\hline 3 & QUV-B $1250 \mathrm{~h}$ & $3 / \mathrm{R}_{2}$ & $7.08 \times 10^{5}$ & 1.680 & 1.110 & 1.620 \\
\hline 4 & 20 h soak & $1 / \mathrm{R}_{1}$ & $8.52 \times 10^{5}$ & 1.910 & 0.908 & 1.650 \\
\hline 4 & $68 \mathrm{~h}$ soak & $2 / \mathrm{R}_{2}$ & $7.23 \times 10^{5}$ & 1.600 & 1.360 & 1.540 \\
\hline 4 & 8 day soak & $3 / \mathrm{R}_{2}$ & $7.07 \times 10^{5}$ & 1.520 & 0.742 & 1.270 \\
\hline 5 & $25 \mathrm{~h}$ soak & $2 / \mathrm{R}_{2}$ & $7.42 \times 10^{5}$ & 1.610 & 0.793 & 1.320 \\
\hline 5 & 7 day soak & $2 / \mathrm{R}_{2}$ & $3.50 \times 10^{5}$ & 0.538 & 0.606 & 0.525 \\
\hline 5 & 62 day soak & $2 / R_{2}$ & $5.35 \times 10^{5}$ & 1.270 & 1.260 & 1.280 \\
\hline
\end{tabular}

*multiple point defects, soak $=$ immersion in electrolyte, $\$$ denotes samples that were incorrectly categorized by that method. 


\section{A.1.2 Discussion}

A cursory inspection of the plots and correlation coefficients in Figure A.1 shows that none of these six methods produced well-defined categories (showing scattering, rather than clustering of data), nor did they display strong correlation with the more traditional method of circuit modeling (displaying poor linearity). This suggests that these methods are overly simplified and that they assume resistance should decrease while capacitances should increase, leading to an overall decrease in impedance in failing coatings. That the methods lack defined categories is not surprising considering that electrochemical features of failing coatings such as the contribution of the double layer and charge transfer resistance can lead to an increase in impedance.

Specifically contributing to the poor correlation seen, the area integration (Fig. A.1A) is weighted toward higher frequencies, where coating capacitance usually dominates and where defects in the coating are not typically observed. If a small defect is present, the effect of that defect on the $\mathrm{Z}$ modulus area would be negligible, since it typically presents at lower frequencies. Spectral features such as these can lead to the mischaracterization of the protective quality of coatings.

Calculating the percentage decrease in the $\mathrm{Z}$ modulus, as shown in A.1B, requires EIS acquisition at two different time points and thus this method is only relative to the starting condition of the coating. The decreasing percentage of the $\mathrm{Z}$ modulus area is $0 \%$ for coatings at their initial timepoints but their initial protective qualities cannot be determined. Alternatively, the area of the phase plot was calculated and plotted against the impedances obtained from circuit modeling (as can be seen in 
Figure A.1C). This plot also shows a poor correlation across all coating types. High frequencies are weighted heavily in the phase area in the same way as the $\mathrm{Z}$ modulus area calculation, masking the influence of defects (when present).

Ratios of the $\log$ of the impedance magnitude did not produce strong correlations or clustering of data when plotted against the circuit modeling results at any of the three frequency ranges investigated (as can be seen in Figure A.1D-F). The low frequency ratio in Figure A.1F, $R l$, had the highest correlation with the impedance values calculated, yielding an $\mathrm{R}^{2}=0.606$.

The negative results from the poor correlation between the previously published methods versus the impedances extracted from traditional circuit modeling emphasize the importance of the positive results presented in the main body of the paper.

\section{A.2 Kramers-Kronig transformations}

Kramers-Kronig transformations are commonly used to validate EIS data. Kramers-Kronig transformations were performed on spectra from intentionally damaged and weathered Paraloid ${ }^{\mathrm{TM}}$ B-44 coatings using Gamry Echem Analyst. Errors were reported in the form of Gamry's goodnes of fit values and can be seen in Figures A.2 and A.3 along with the transformations. The spectra having the largest (poorest) values were taken without the use of a Faraday cage, which had values on the order of $10^{-4}$. All other EIS spectra gave excellent matches (having small errors, on the order of $\left.10^{-5}-10^{-6}\right)$ and thus demonstrate the validity of these EIS data sets. 
A)

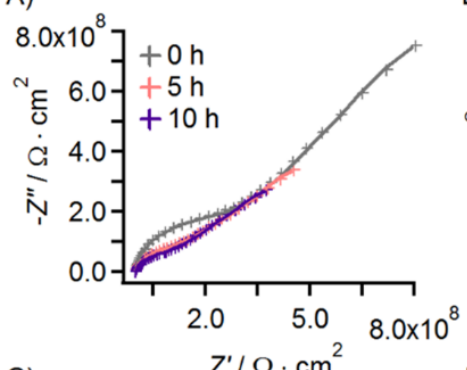

C)

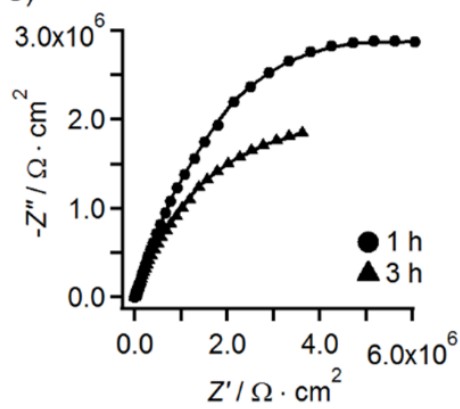

B)

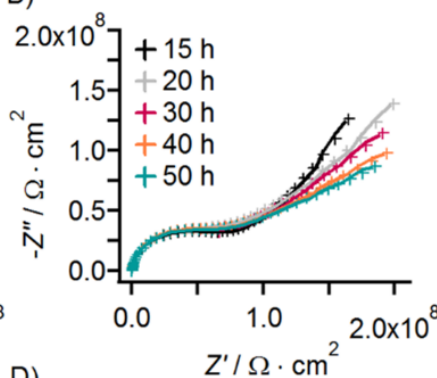

D)

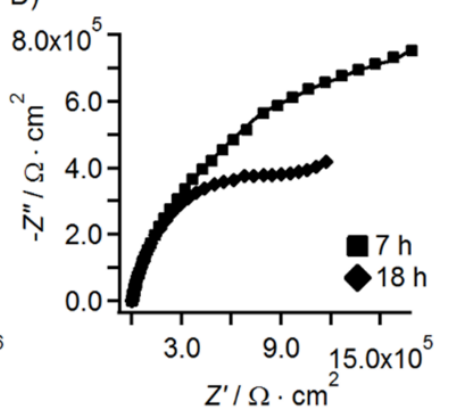

Figure A.2 Nyquist plots (data points) and Kramers-Kronig transformations (as fit lines) of Figures 3A$\mathrm{B}$ and 4 and corresponding goodness of fit values shown in the table to the right of the graphs: A-B) Figures 3A-B; an abraded Paraloid ${ }^{\text {TM }}$ B-44 coating immersed in electrolyte for 50 hours and C-D) Figure 4; a Paraloid ${ }^{\mathrm{TM}} \mathrm{B} 44$ coating with multiple point defects immersed in electrolyte for 18 hours

A)

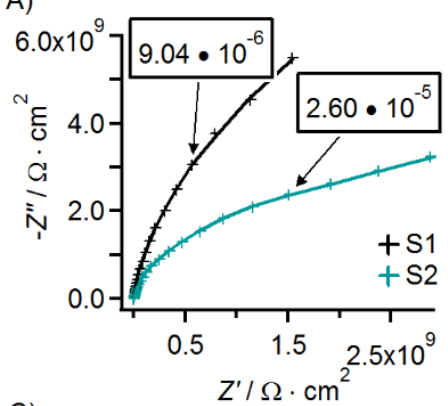

C)

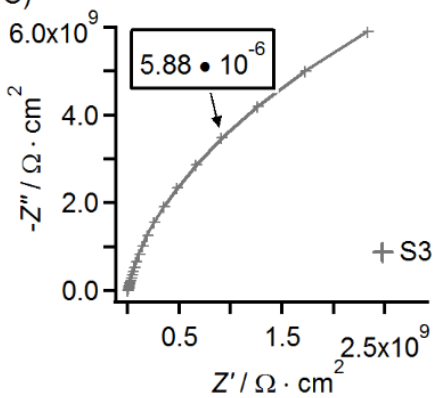

B)
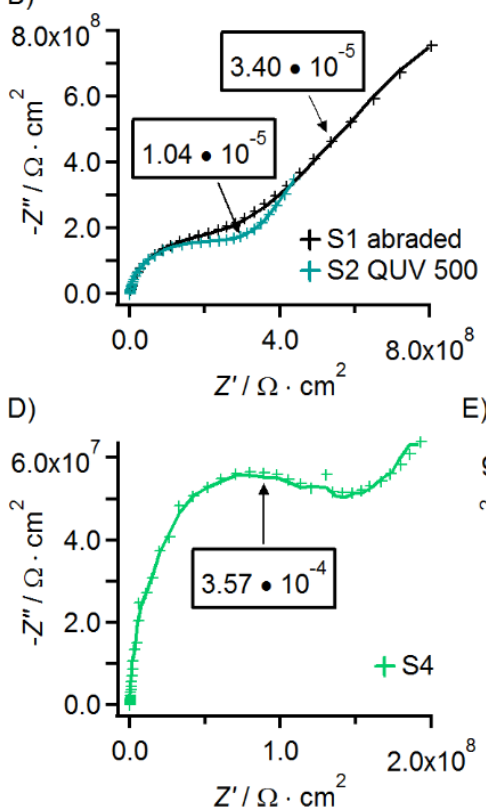

E)

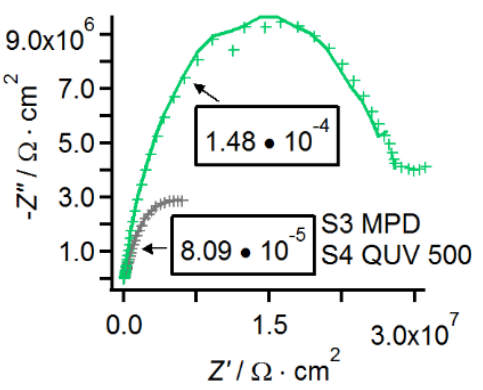

Figure A.3 Nyquist plots (data points) and Kramers-Kronig transformations (as fit lines) of Figure 1.5 and corresponding goodness of fit values are inset: A) S1 and S2 before damage, B) S1 and S2 after damage, C) S3 before damage, D) S4 before damage, and E) S3 and S4 after damage. 